\title{
Complutum
}

ISSN: 1131-6993

\section{A propósito del poblamiento aborigen en Gran Canaria. Demografía, dinámica social y ocupación del territorio ${ }^{1}$}

\author{
Javier Velasco-Vázquez², Verónica Alberto-Barroso ${ }^{3}$, Teresa Delgado-Darias ${ }^{4}$, Marco Moreno-Benítez
}

Recibido: 01/03/2021 / Aceptado: 04/05/2021

Resumen. Este trabajo aborda el estudio de la dinámica poblacional y demográfica de la ocupación prehispánica de Gran Canaria. Para ello se ha recurrido al análisis de la distribución del sumatorio de probabilidad de las dataciones radiocarbónicas procedentes, en este caso, de contextos funerarios. Los resultados obtenidos se compararon con dos modelos teóricos nulos de crecimiento poblacional, tratando de explicarlos teniendo en cuenta la información arqueológica disponible, en relación con los cambios diacrónicos registrados en las fórmulas sepulcrales indígenas, así como a partir de las variaciones detectadas en la ocupación del territorio a lo largo de todo el poblamiento insular. Para precisar el marco temporal sujeto a examen, se llevó a cabo un análisis estadístico formal estimativo del intervalo de confianza para establecer la fecha de poblamiento más temprana de Gran Canaria. Se contribuye también de este modo al renovado debate sobre las fechas de los primeros establecimientos permanentes de población en el Archipiélago. Todo ello ha facilitado una propuesta de marco temporal y social desde el que explicar las particularidades de un proceso de poblamiento insular complejo en el que confluyen dinámicas internas y externas.

Palabras clave: distribución de sumatorio de probabilidad; Gran Canaria; poblamiento insular; demografía; cronología.

\section{[en] Revisiting the process of aboriginal population of Gran Canaria. Demography, social dynamics and occupation of the territory}

\begin{abstract}
This work addresses the study of the population and demographic dynamics of the pre-Hispanic occupation of Gran Canaria. For this purpose, we have resorted to the analysis of the summed probability distributions of radiocarbon dates from different funerary contexts. The results were compared with two theoretical null models of population growth, explaining them based on the available archaeological information, linked to the diachronic changes recorded in the indigenous burials, as well as the variations in the occupation of the territory throughout the historical sequence of the ancient Canarians. To specify the time frame under examination, a formal statistical analysis was carried out to estimate the confidence intervals to place the earliest date of settlement of Gran Canaria. This work also contributes to the renewed debate on the dates of the first permanent settlements in the archipelago. In sum, this has facilitated the proposal of a temporal and social framework to explain the particularities of a complex process of island population in which internal and external dynamics converge.
\end{abstract}

Keywords: Summed probability distributions (SPD); Gran Canaria; Island occupation; demography; chronology.

Sumario: Introducción. 1. Material y método. 2. Resultados. 3. Discusión. 3.1. Los inicios del poblamiento efectivo de Gran Canaria. 3.2. Dinámica demográfica. 4. Conclusiones. Bibliografía. Anexo.

Cómo citar: Velasco-Vázquez, J.; Alberto-Barroso, V.; Delgado-Darias, T.; Moreno-Benítez, M. (2021): A propósito del poblamiento aborigen en Gran Canaria. Demografía, dinámica social y ocupación del territorio. Complutum, 32(1): 167-189.

\footnotetext{
Este trabajo se inserta en el Proyecto "Cuerpos, objetos y espacios. Muertes convergentes, muertes divergentes" (2018PATRI05) financiado con fondos para investigación de la Fundación CajaCanarias y la Fundación Bancaria La Caixa.

2 Servicio de Patrimonio Histórico del Cabildo de Gran Canaria. Calle Bravo Murillo, 23, 35002, Las Palmas de Gran Canaria. Grupo de Investigación TARHA. Universidad de Las Palmas de Gran Canaria.

E-mail: jvelascov@grancanaria.com

3 Tibicena. Arqueología y Patrimonio.

4 El Museo Canario.

5 Tibicena. Arqueología y Patrimonio.
} 


\section{Introducción}

La reciente propuesta de cambios diacrónicos en las fórmulas funerarias de las antiguas poblaciones de Gran Canaria (Alberto et al., 2019) y su estrecha relación con otros factores de dinámica social: estrategias económicas, políticas, ocupación del territorio, procesos migratorios, etc. (Alberto et al., 2020, Moreno, 2020) ha llevado a desechar la idea de una ocupación prehispánica de la isla como un todo heterogéneo, con unas manifestaciones culturales que apenas se modificarían en el transcurso de casi un milenio y medio. Además, en los últimos años, otros trabajos han registrado cambios puntuales en algunas expresiones arqueológicas, circunscritos a los últimos siglos de esta secuencia histórica que se explican como resultado de procesos internos debidos básicamente al incremento de población (Velasco, 2018; Morales, 2019). No obstante, y hasta la propuesta de caracterización y ordenación de la práctica funeraria, han faltado las perspectivas de más largo alcance desde las que evaluar el poblamiento aborigen como una realidad mutable, dinámica, con cambios y permanencias a lo largo tiempo. La información disponible, las renovadas perspectivas en la forma de entender este pasado y la disponibilidad de nuevas herramientas de análisis, invitan a afrontar el examen a largo plazo de algunos de los procesos sociales que se dan en la isla. En este caso, entendiendo el tiempo como una estrategia de análisis social y no como un mero marco de referencia estático en el que ir situando determinados elementos del registro arqueológico.

El análisis diacrónico de las dinámicas sociales puede valerse de algunas de las herramientas de la paleodemografía, pues se trata de una variable a la que tradicionalmente se otorga un destacado protagonismo como agente del cambio cultural. El análisis demográfico ayuda a entender modificaciones en las prácticas de subsistencia, la complejidad social, los conflictos intergrupales, los patrones de ocupación del territorio, etc. (Puleston y Winterhalder, 2019; Palmisano et al., 2020). La generación de modelos con capacidad para precisar las dinámicas de las poblaciones del pasado, así como el examen de las causas que motivan su estabilidad o inestabilidad, contribuye a ofrecer una explicación histórica de los cambios culturales identificados en el registro arqueológico, en una amplia variedad de escalas cronológicas y territoriales. En este sentido, los contextos insulares representan un marco ideal desde el que investigar estas relaciones a largo plazo: tendencias demográficas/dinámicas sociales y las fórmulas culturales identificadas en estos ámbitos. En especial, en casos como el de Gran Canaria, con unas dimensiones reducidas (poco más de $1500 \mathrm{~km}^{2}$ ), un marco temporal acotado (menos de 1500 años) y donde los contactos con el exterior (probablemente, eventos de llegada de población) parece que fueron capaces de introducir cambios sustanciales en ciertos momentos (Alberto et al., 2020), pero en apariencia como consecuencia de episodios puntuales.

En esta aproximación al modelo aborigen grancanario es importante precisar el margen temporal en el que situar el análisis, sobre todo en lo que se refiere al inicio de la secuencia considerada, pues su final se marca con claridad a finales del siglo XV d.C. con la conquista europea. El comienzo del poblamiento o más precisamente del establecimiento permanente de población norteafricana en el archipiélago, y en particular en Gran Canaria, ha sido objeto de un amplio debate que parece clarificarse en los últimos años a partir, fundamentalmente, de la información ofrecida por el C14. Sin embargo, la controversia sigue abierta y la flexibilidad en la forma de utilizar las dataciones continúa provocando que el límite inferior para ese primer poblamiento oscile, principalmente, entre la primera mitad del primer milenio a.C. y los tres primeros siglos de la Era.

En este trabajo se propone un enfoque estadístico formal para estimar los intervalos de confianza en los que situar el poblamiento más temprano. Todo ello asumiendo la premisa de que el contexto arqueológico con la fecha absoluta más antigua conocida no será, con toda probabilidad, el más antiguo de Gran Canaria, en función del sesgo tafonómico de los sitios de la primera ocupación (Crema et al., 2017; Prates et al., 2020; Porčić et al., 2021). Sin embargo, con este ensayo se busca estimar un límite cronológico confiable y estadísticamente bien respaldado (Prates et al., 2020) para el análisis de toda la secuencia. Lógicamente, a medida que se avance en la investigación, esta información puede ser objeto de revisión, pero necesariamente las valoraciones se deben sustentar en datos verificados y fiables para el planteamiento de nuevas hipótesis de trabajo. 
En los últimos años, el estudio de dinámicas poblacionales ha experimentado un auge destacado, sobre todo a partir del empleo del análisis del sumatorio de las distribuciones de probabilidad de las dataciones radiocarbónicas (en adelante SPD en su acrónimo anglosajón). De forma resumida, los supuestos básicos de este método serían, por un lado, la existencia de una correlación entre el tamaño de la población y la cantidad de restos materiales que esta generaría y, por otro, que la muestra de restos materiales utilizados para la datación es representativa del conjunto. Ello implica que la distribución de frecuencia de fechas absolutas debería reflejar cambios en el tamaño relativo de la población, más precisamente de la dinámica poblacional, en un intervalo de tiempo concreto (Porčić et al., 2021). Con todo, es un procedimiento que ha sido objeto de críticas, en especial por los sesgos esperables en las fechas disponibles. Ya sea por las trayectorias de la investigación arqueológica en cada lugar, la mayor probabilidad de subrepresentación de los enclaves más antiguos o por otros factores que también pueden intervenir en el resultado de este análisis, como son los cambios en los sistemas productivos, las características de la curva de calibración, tamaño de la muestra, heterogeneidad del tipo de materiales analizados, etc. (Contreras y Meadows, 2020). Aunque buena parte de estos problemas cuentan ya con fórmulas para minimizar su influencia en un análisis global (Cremay Kobayashi, 2020) se trata de variables que pueden introducir "ruido" en la aplicación del método sin que sea posible recurrir a una receta general que garantice la idoneidad absoluta de su examen (Carleton et al., 2020). Pese a todo, la SPD es una herramienta de análisis práctica y en cuya valoración particular es imprescindible introducir la mayor cantidad de información arqueológica disponible para dar sentido histórico a los datos obtenidos.

En Gran Canaria, salvo una referencia puntual (Pardo y Barceló, 2020: 491), esta cuestión no ha sido abordada como fórmula para la elaboración de una propuesta sobre la dinamia del poblamiento aborigen y la valoración de qué factores (internos y externos) pudieron estar interviniendo en su particular desarrollo.
Existe un consenso general, aunque sin alcanzar unanimidad en la cifra de habitantes, en señalar a esta isla como la más poblada del Archipiélago en el momento de la conquista (Jiménez, 1999; Onrubia, 2003; Mederos, 2019). No obstante, es muy poco lo que se conoce sobre la situación previa, más aún si se incorpora una perspectiva a largo plazo de esta variable en el desarrollo histórico de los antiguos canarios. Los procedimientos tradicionales de estimación poblacional propuestos o ensayados no han proporcionado resultados que permitan ser optimistas (Onrubia, 2003), lo que ha llevado a que todos los esfuerzos se focalizaran en una imagen postrera de un proceso que sigue siendo desconocido.

En este marco, el presente trabajo constituye una aproximación general a esta materia, en un intento de aportar información contrastada desde la que construir una lectura global del proceso de poblamiento, con capacidad para reconocer dinámicas sociales particulares, su origen y vigencia, así como para detectar procesos de cambio.

\section{Material y método}

Para este trabajo se han seleccionado un total de 171 dataciones radiocarbónicas, de 43 yacimientos arqueológicos de Gran Canaria (Fig. 1), todas ellas procedentes de contextos mortuorios. La mayor parte de las fechas ya han sido objeto de publicación en trabajos previos (Alberto et al., 2019; Velasco et al, 2020), a las que ahora se añaden algunas inéditas que completan la serie (véase Anexo: Tabla 1). Todas ellas responden a materiales "de vida corta", sin aparentes problemas de contaminación o degradación y no cuestionadas en su publicación original. Por razones conocidas se ha prescindido de las fechas procedentes de contextos funerarios realizadas sobre carbón o madera sin determinación específica $y$, en particular, las procesadas en el laboratorio de Gakushuin por sus demostrados problemas de fiabilidad y precisión (Blakeslee, 1994; Mulrooney, 2013; DiNapoli et al., 2020; Quintus et al., 2020; para el caso concreto de Canarias: Velasco et al., 2020). 


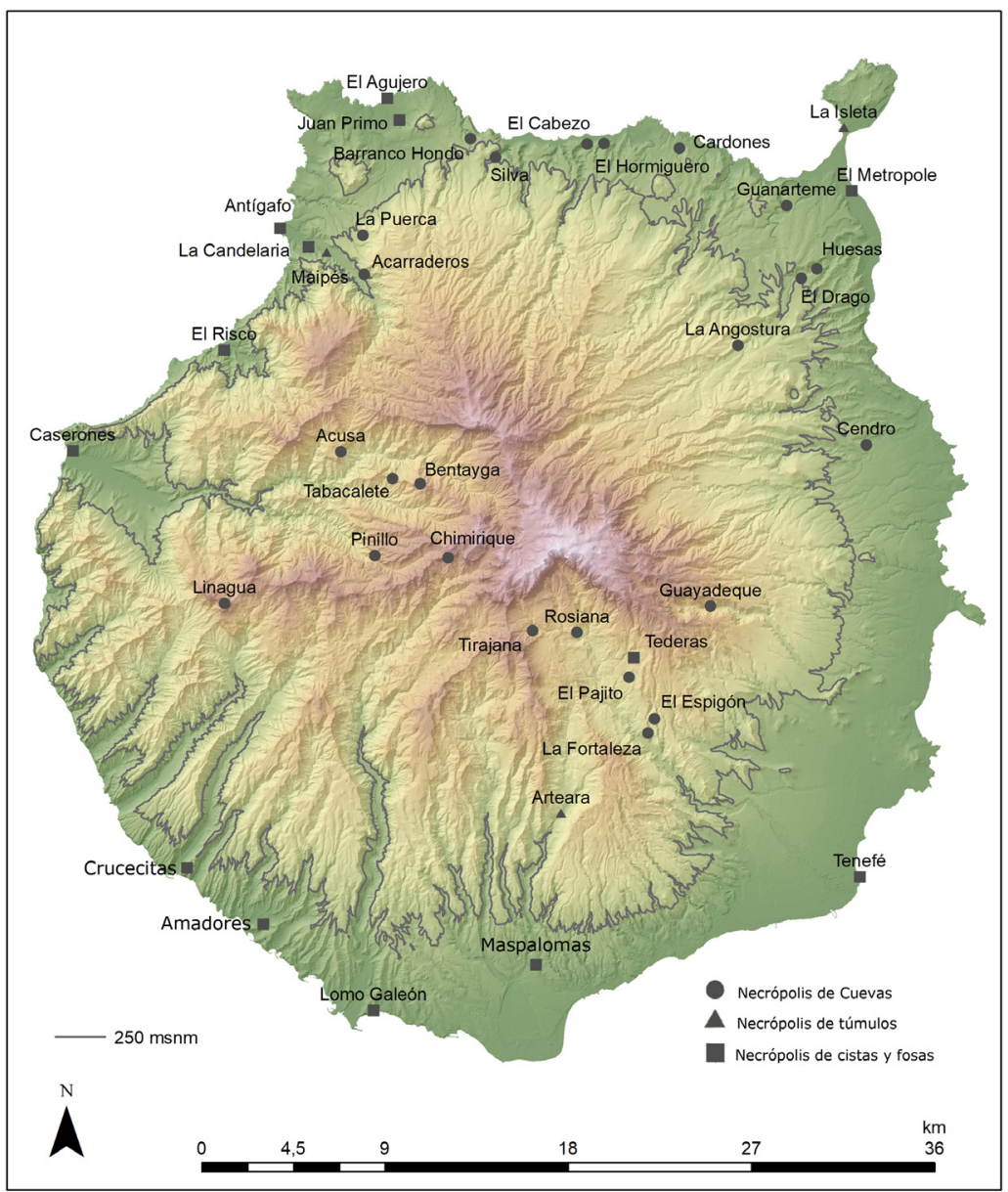

Figura 1: Localización de los yacimientos citados e indicación de la línea de los 250 msnm.

Dados los objetivos planteados se podía haber seleccionado un número más elevado de fechas, incorporando también las correspondientes a contextos domésticos, lo que hubiera permitido sumar algo más de medio centenar de dataciones al estudio. Sin embargo, cualquier propuesta de modelización de la dinámica poblacional a largo plazo pasa también por identificar aquellos materiales arqueológicos capaces de proporcionar las medidas indirectas de mayor confianza y excluir aquellas que pudieran estar condicionadas por otros factores ajenos a las variables objeto de estudio. Siempre desde la premisa que tales indicadores no ofrecen la evidencia directa del número absoluto de personas en el pasado, sino una estimación de las intensidades relativas de la población y del cambio a lo largo del tiempo (Palmisano et al., 2017). Por estas razones, la opción de introducir las fechas de contextos domésticos supondría asumir un muestreo con problemas de partida. En primer lugar, hubiera supuesto incorporar muestras de distinta naturaleza, presentes en los yacimientos como resultado de actividades humanas diversas y, en ocasiones, no bien conocidas, asumiendo con ello incertidumbres que no siempre pueden valorarse en su justa medida. En segundo lugar, y como principal razón, la abrumadora disposición de fechas situadas en el segundo milenio, atribuible sobre todo a la trayectoria investigadora de las últimas décadas en la arqueología grancanaria. Si hacemos un balance de las dataciones de contextos domésticos recopiladas en un trabajo reciente (Velasco et al., 2020) que cumplan unas condiciones mínimas de higiene radiométrica, menos del $15 \%$ correspondería al primer milenio de la era, situándose preferentemente a partir del siglo IX. Frente a ello, los contextos mortuorios tienen la ventaja de abarcar la secuencia cronológica completa, con una distribución menos parcial, proceder de sepulturas prima- 
rias y en un porcentaje muy elevado de los casos (más del 90\%) directamente obtenidas de los propios restos humanos. Asimismo, las muestras de mortajas funerarias proporcionan un grado de certidumbre apropiado, pues no constituyen agregados y en los casos en los que se ha fechado el fardo y los restos humanos asociados, los resultados no presentan diferencias estadísticamente significativas (Alberto et al., 2019; Alberto 2020).

Es cierto que con los criterios expuestos, el número de muestras es relativamente bajo y no llega al mínimo recomendado por algunos autores para el desarrollo de trabajos como el que se propone, lo que a priori introduce una cierta debilidad en los resultados (Williams, 2012). Sin embargo, no existe un umbral mínimo aplicable a todos los casos, pues la validez del tamaño de la muestra dependerá también de los objetivos y del alcance del análisis (Crema y Bevan, 2020). En este trabajo, el número de dataciones está cerca de las 200 consideradas un mínimo aceptable por algunos autores, a lo que se suma que se aborda el estudio de un ámbito geográfico con una extensión territorial sensiblemente inferior a la de otros trabajos en esta línea y un marco cronológico relativamente reducido. Todo ello permite partir de la premisa de una representatividad global admisible. De cualquier modo, se asume que los resultados deben considerase provisionales y con la posibilidad de cambios al incrementar los datos radiométricos (Williams, 2012; Contreras y Meadows, 2020; Aubán et al., 2018). La valoración de esta información en un marco histórico concreto ayudará a precisar la fiabilidad, precisión y alcance de la información obtenida (Crema y Bevan, 2020; Hinz, 2020).

Para abordar el enfoque estadístico formal capaz de estimar los intervalos de confianza para la población más temprana de Gran Canaria y obtener el patrón temporal del extremo más antiguo de la distribución de fechas calibradas se ha seguido el procedimiento empleado por Di Napoli y colaboradores (2020; véase también Ramsey, 2009). Según este modelo de estimación, se seleccionaron las diez dataciones más antiguas disponibles para Gran Canaria, todas ellas de contextos funerarios, agrupándolas como si fueran una única fase, entendiendo que su límite de inicio proporcionaría una estimación del comienzo del poblamiento estable de la isla. No se han incorporado otras fechas radiocarbóni- cas procedentes de contextos no sepulcrales que proporcionan dataciones algo más antiguas que las disponibles, pues no cumplen las condiciones básicas de higiene radiométrica (carbones no determinados, sin contexto arqueológico definido, agregados, sedimentos...) y muy probablemente introducirían sesgos evidentes.

La creación de las SPD y los análisis posteriores se llevaron a cabo en el entorno $R$ Statistical 4.0.3 (R CoreTeam 2020), utilizando el paquete rcarbon, versión 1.4.1 (Crema y Bevan 2020a; 2020b) y la curva IntCall 20 (Reimer et al., 2020). La distribución de probabilidad sumada generada a partir de las 171 fechas se realizó dentro de una cobertura temporal que va entre 2000 y $200 \mathrm{BP}$, más amplia que nuestro período de estudio, para de este modo evitar efectos de borde. Se trató de minimizar cualquier sesgo de sobremuestreo de fases o eventos cronológicos específicos, agregando fechas de radiocarbono no calibradas dentro de "compartimentos" (bins) de 50 años, como así fue determinado en el análisis de sensibilidad desarrollado (Crema y Bevan, 2020; Timpson et al., 2014).

Se comparó la SPD obtenida de fechas de radiocarbono calibradas con dos modelos teóricos nulos de crecimiento de la población (exponencial y logístico) para determinar estadísticamente si las fluctuaciones de dinámica poblacional inferidas indican desviaciones significativas con respecto a lo que podría suceder por azar (Timpson et al., 2014). La observación de desviaciones significativas positivas y negativas con respecto a los modelos nulos se realizó empleando 1000 simulaciones de Monte Carlo, lo que minimizó el sesgo causado por el proceso de calibración (Roscoe et al., 2021). Las desviaciones de la SPD (línea negra continua en las figuras) por encima y por debajo de la envolvente de confianza indican respectivamente períodos de aumento (remarcado en rojo en sus valores significativos) o disminución (en azul) mayores de lo esperado según el modelo nulo de crecimiento seleccionado. Aunque tanto los modelos logísticos como exponenciales son abstracciones teóricas que no describen ningún crecimiento poblacional derivado empíricamente, son útiles para evaluar patrones significativos en las fluctuaciones poblacionales a lo largo del tiempo. En particular y de cara a justificar esta elección (Crema y Bevan, 2020b), el modelo exponencial, además 
de minimizar la pérdida de información de los episodios de ocupación más antiguos (Timpson et al., 2014), se vincula a los supuestos demográficos más sencillos y que, en su caso, ayudarían a explicar la elevada tasa de población al final de esta secuencia histórica. Por su parte, el logístico contribuiría a asociar los datos de población con las limitaciones de capacidad de carga que pudiera tener, según el modelo económico, un territorio insular como el nuestro.

En este sentido hay que reseñar que, de forma predeterminada, en el paquete rcarbon las probabilidades de las fechas calibradas se normalizan para que la probabilidad total sea igual a uno, como sucede en la mayoría de los programas de calibración de radiocarbono (Crema y Bevan, 2020a). Sin embargo, Weninger y colaboradores (2015) han advertido que cuando las fechas se agregan por suma, este proceso de normalización puede generar picos artificiales en las distribuciones de probabilidad resultantes que coinciden con porciones más pronunciadas de la curva de calibración (Crema y Bevan, 2020b). El uso de calibraciones normalizadas o no normalizadas no tiene un impacto en la forma de cada distribución de probabilidad calibrada de fechas individuales, pero sí influye en la forma de la SPD. Para explorar estas diferencias en nuestros resultados se generaron curvas con fechas normalizadas y no normalizadas, como así se recomienda en los trabajos de referencia (Crema y Bevan, 2020b).

En un intento de buscar otros elementos explicativos sobre la tendencia de las curvas de la SPD comparadas con los modelos nulos, se construyeron secuencias teniendo en cuenta los contextos arqueológicos de procedencia de las muestras, en concreto la tipología de la sepultura (Fig. 2). Así, las variaciones observadas en la práctica funeraria se han relacionado con cambios significativos en el patrón de ocupación de la isla, en las estrategias económicas y el grado de jerarquía social, lo que quizá ayudaría a entender las oscilaciones en la curva global.

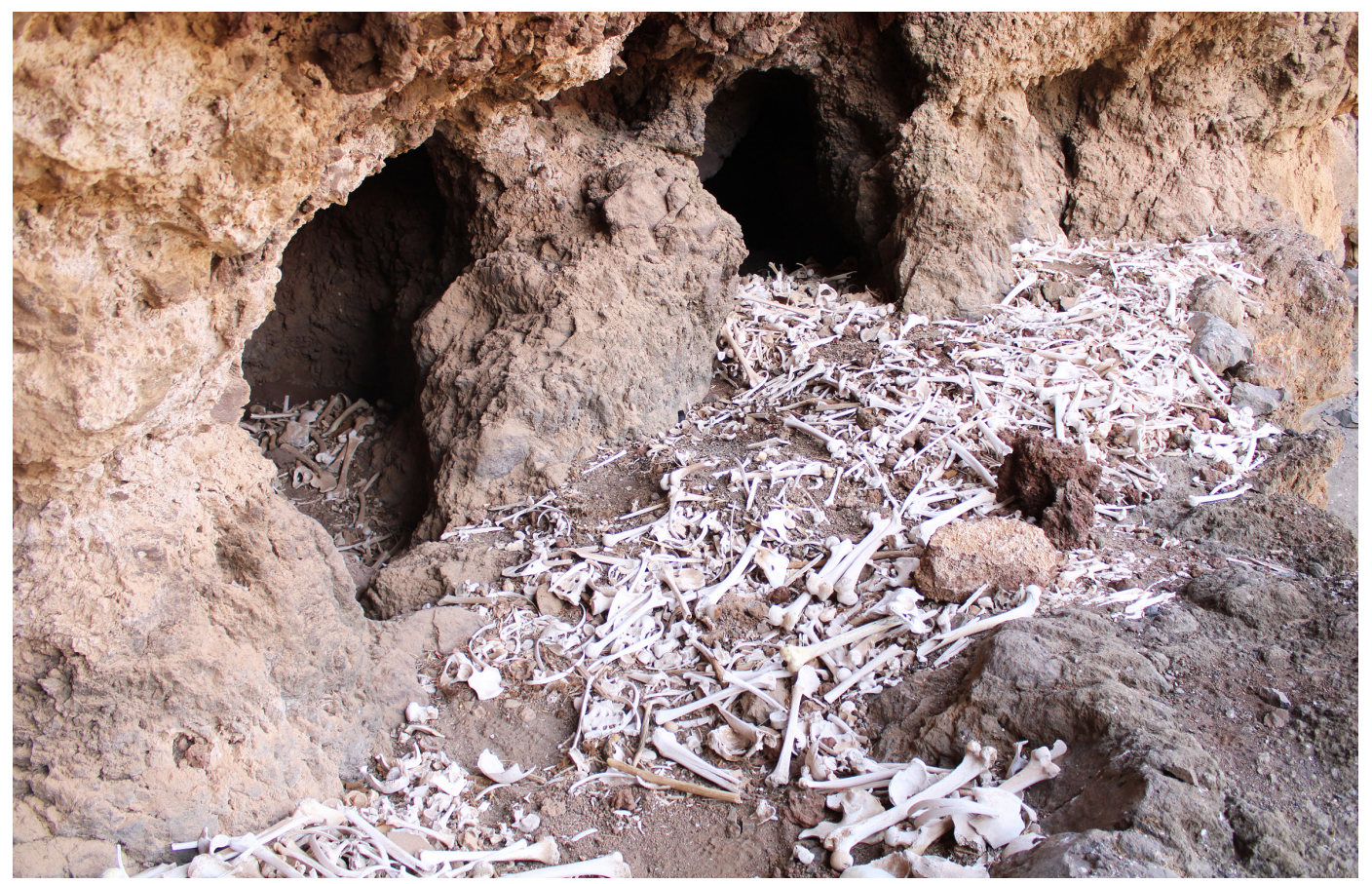

Figura 2: Sepultura en cueva. Cementerio de los Canarios (Bco. de Guayadeque, Agüimes).

También se ha intentado establecer comparaciones en relación con la localización de los enclaves muestreados, si bien en un territorio de dimensiones tan limitadas y abrupta topografía como el de Gran Canaria esta es una variable compleja y difícil de objetivar. Para ello se han distinguido los yacimientos ubicados hasta los $250 \mathrm{msnm}$ aproximada- 
mente de aquellos situados por encima de esa cota (Fig. 1). Es evidente que se trata de una división artificial y que, en último término, precisaría mayores matizaciones, pero puede ser un primer paso para abordar las estrategias de ocupación del territorio en esta secuencia histórica. A grandes rasgos, los lugares de la primera categoría, la franja inferior, se caracterizan por su ubicación en terrenos llanos, con mejores condiciones agrícolas y por la inmediatez de los recursos del litoral. El segundo (por encima de los $250 \mathrm{msnm}$ ) se asociaría a una topografía más escarpada, con menor disponibilidad de suelos aptos para la agricultura, pero con un mayor potencial ganadero (Moreno y González, 2016).

\section{Resultados}

Para el inicio de la colonización efectiva de Gran Canaria el modelo ensayado estima que este proceso se inicia en el rango 233-435 cal. d.C. $\left(\mathrm{A}_{\text {model }}=104.9 ; \mathrm{A}_{\text {overall }}=104.2\right)$. Se tra- ta de un margen amplio, que en su extremo inferior podría situarse en el primer tercio del siglo III d.C., envejeciendo con ello la fecha que proporcionan las dataciones fiables existentes para Gran Canaria. En todo caso, es muy probable que esta estimación deba retrasarse algo más en el tiempo pues las cronologías de los primeros momentos tienen menos probabilidades de estar bien representadas en la secuencia. Esta estimación en torno a principios del III d.C. es un dato coincidente con las fechas disponibles para el resto de las islas.

La información obtenida a partir de los gráficos de SPD muestra un modelo de dinámica poblacional coherente con la información arqueológica. La SPD de las fechas en su conjunto (Fig. 3) marca una tendencia de crecimiento progresivo, más pausado en sus primeros momentos que luego tiende a estabilizarse, e incluso descender ligeramente, para más tarde incrementarse de forma significativa.

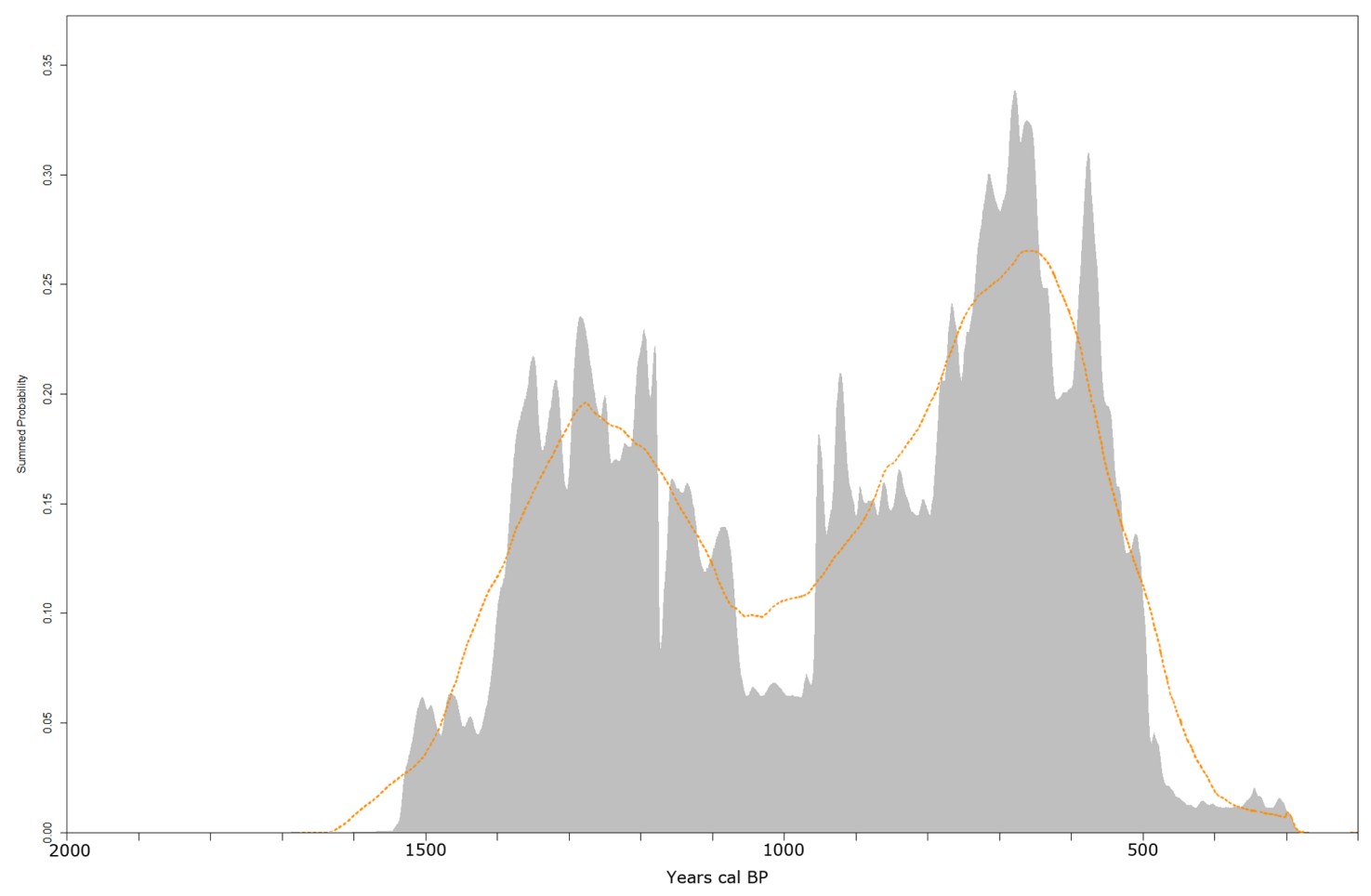

Figura 3: Gráfica de distribución del sumatorio de probabilidad de las fechas radiocarbónicas (SPD).

Esta trayectoria es aún más clara atendiendo a la gráfica SPD generada según las agrupaciones de bins de 50 años (Crema y Bevans, 2020a). En este último caso (Fig. 4), 
el modelo es prácticamente idéntico, aunque algunas variaciones son menos acusadas, mostrando un patrón de crecimiento paulatino y posterior estabilización en buena parte de la secuencia contemplada. Esta tendencia sufre un cambio significativo en torno al
800-700 BP (1150-1250 d.C.), en el que el incremento es mucho más acusado en un período de tiempo breve, para luego descender de forma muy acusada, también en un lapso temporal corto.

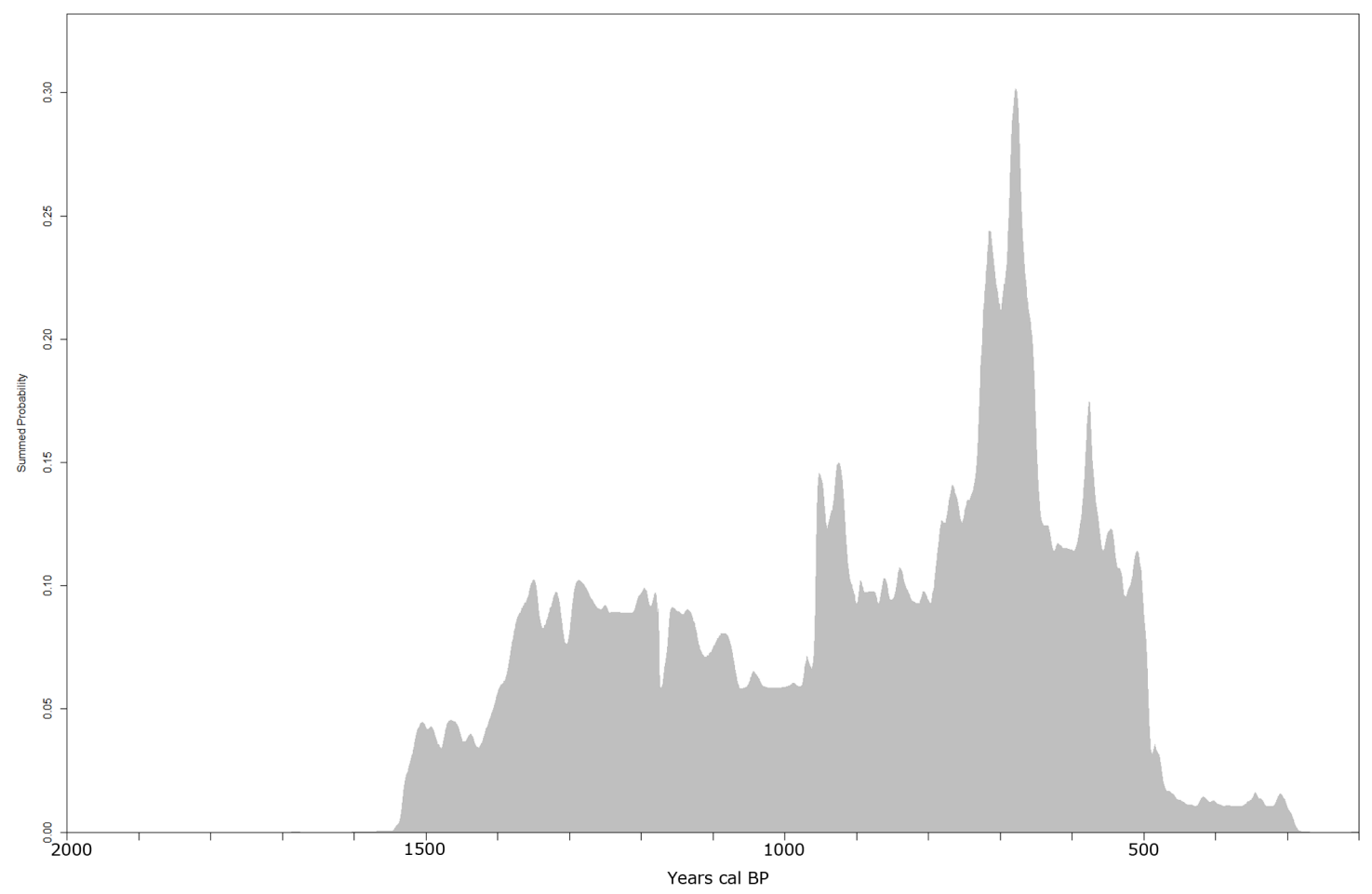

Figura 4: Gráfica de distribución del sumatorio de probabilidad de las fechas radiocarbónicas (SPD), agrupadas en bins de 50 años para evitar sobrerepresentaciones.

Para evitar el sesgo que podría generar la lectura directa de estas gráficas (Brown y Crema, 2019; Crema y Bevans, 2020b), la distribución de la suma de probabilidad se comparó con dos modelos teóricos de crecimiento poblacional (exponencial y logístico). Estos modelos de hipótesis nula fueron generados, como ya se indicó, con fechas normalizadas y no normalizadas.

Las diferencias en las curvas de la SPD con fechas normalizadas y no normalizadas son apreciables tanto en el modelo nulo exponen- cial (Fig. 5) como en el logístico (Fig. 6), si bien en ambos casos el perfil general se mantiene, acentuándose o reduciéndose su curva en zonas puntuales. Por ello no debe extrañar que los resultados del análisis de la SPD con respecto a los modelos nulos sean parecidos. En todo caso, alcanzando mayor significación estadística cuando se trata de fechas normalizadas. En todos ellos se observan desviaciones positivas y negativas con significación estadística en unos intervalos cronológicos semejantes. 

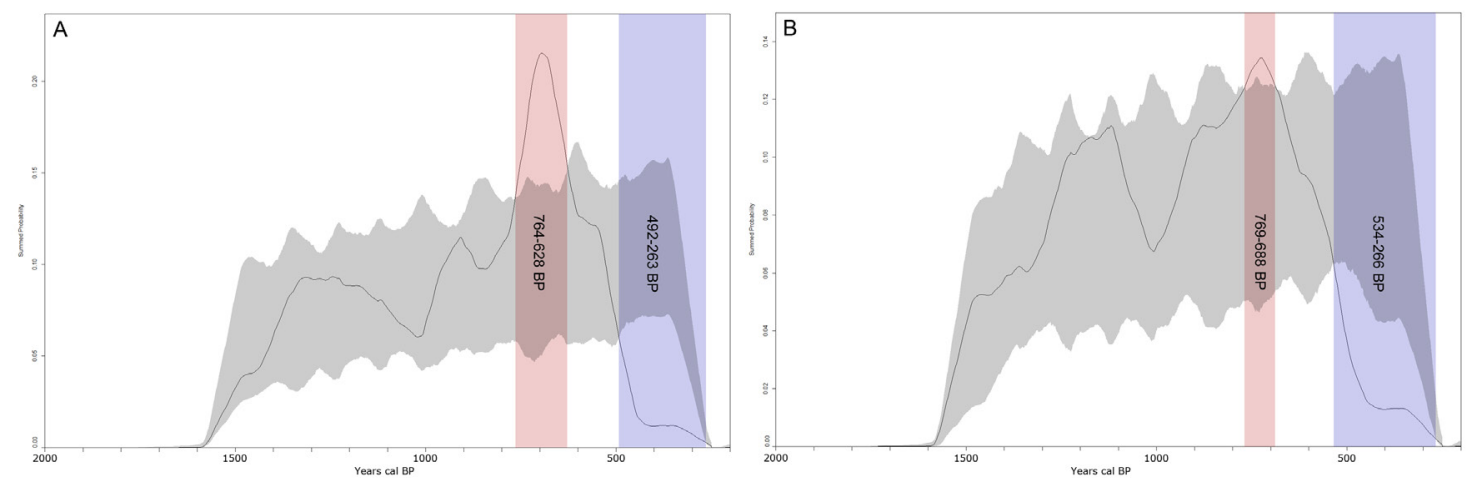

Figura 5: Modelo nulo exponencial. Número de dataciones: 171. Número de bins: 107. Significación estadística empleando 1000 simulaciones. Fechas normalizadas (A): $p=0,001$. Desviación significativa positiva: 764-628 BP; desviación significativa negativa: 492 263 BP. Fechas no normalizadas (B): $\mathrm{p}=0,01598$. Desviación significativa positiva: 769-688 BP; desviación significativa negativa: 534-266 BP.
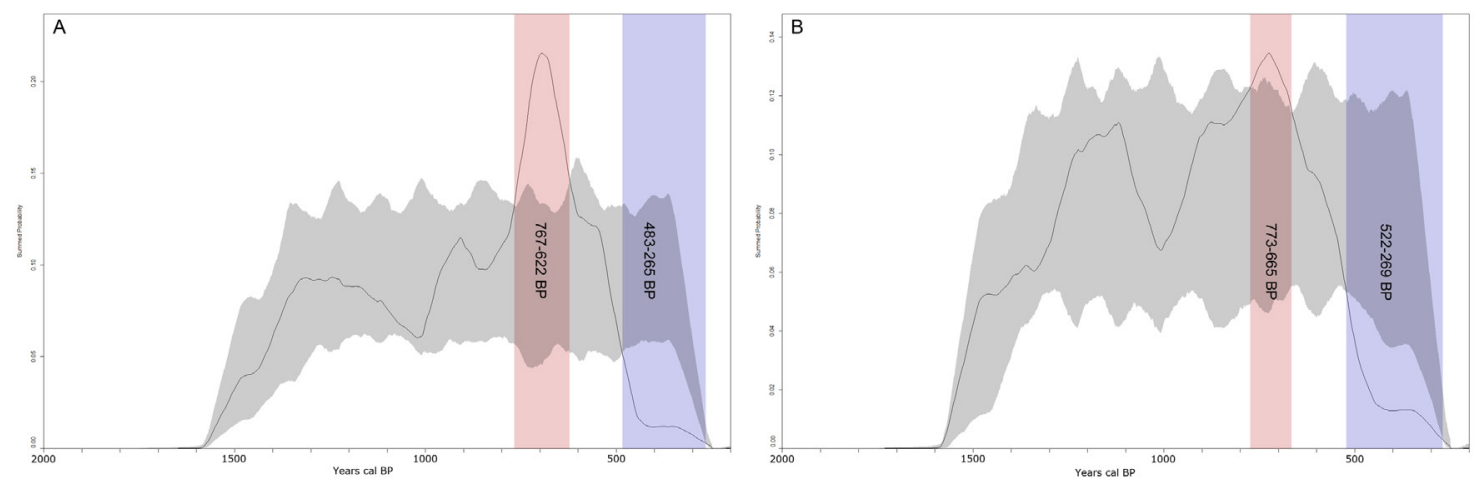

Figura 6: Modelo nulo logístico. Número de dataciones: 171. Número de bins: 107. Significación estadística empleando 1000 simulaciones. Fechas normalizadas (A): $p=0,004$. Desviación significativa positiva: 767-622 BP; desviación significativa negativa: 483-265 BP. Fechas no normalizadas (B): p=0,02198.

Desviación significativa positiva: 773-665 BP; desviación significativa negativa: 522-269 BP.

Hay que destacar que hacia el final de la secuencia histórica analizada se marca un importante ascenso de la curva que, grosso modo, podría situarse en el intervalo 800650 BP (1150-1300 d.C.), para luego iniciar un descenso súbito que alcanza significación estadística aproximadamente a partir del 500 BP (1450 d.C.). Por su parte, en la primera mitad de la secuencia, aproximadamente hasta el 1000 BP (950 d.C.), no se observan crecimientos o decrecimientos significativos con respecto a los modelos nulos, lo que quizá sea reflejo de un progreso poblacional moderado que a priori parece ajustarse algo más a un modelo logístico. En líneas generales, se puede mantener la propuesta de una tendencia global de episodios de crecimiento a los que siguen otros de mayor estabilidad o ligero descenso, al menos en el curso del primer mi- lenio de la era y que se modifica luego a partir del segundo.

$\mathrm{Si}$ valoramos las diferentes curvas de la SPD atendiendo a la tipología del soporte fúnebre (Fig. 7), el primer aspecto a destacar, y sobre el que ya se advertía en trabajos previos, son las diferencias cronológicas en el momento de aparición de cada una de estas manifestaciones, lo que ayuda precisar el alcance de los resultados presentados, más aún cuando tales cambios se pueden asociar también a modificaciones en las estrategias económicas y ocupación del territorio (Alberto, et al., 2020). Si comparamos cada serie de fechas por tipología con su propio modelo nulo, ninguna de ellas alcanza significación estadística global y tan solo reflejan cambios significativos en directa asociación con los máximos alcanzados en su particular desarrollo histórico: las cuevas en el 
período 1377-1287 BP (cal. 573-663 d.C.), los túmulos entre 1069-943 BP (cal. 881-1007
d.C.) y las fosas-cistas entre 602-522 BP (cal. 1348-1428 d.C.).

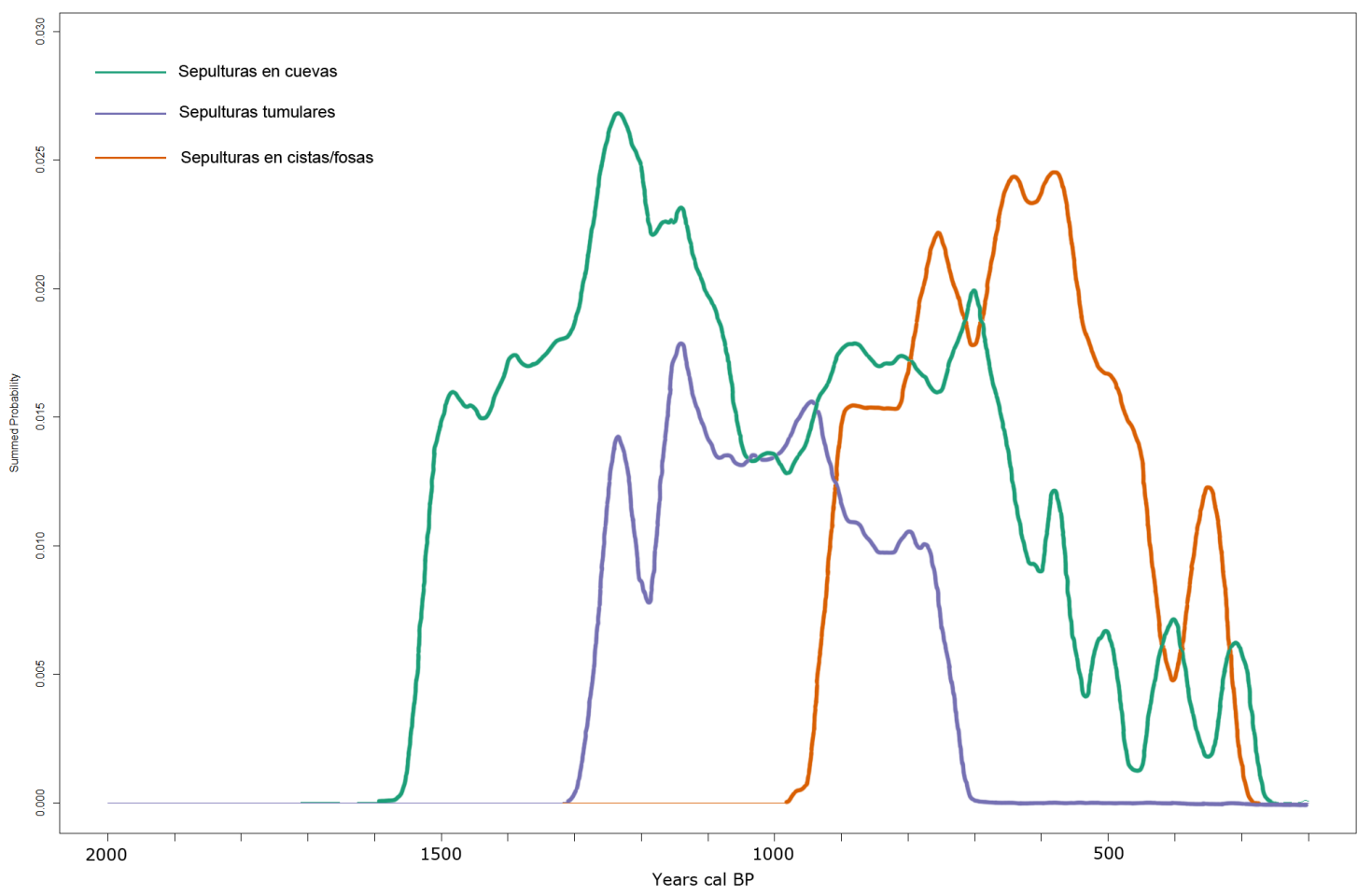

Figura 7: Distribución de suma de probabilidad por tipología funeraria.

La distinción territorial de la procedencia de las muestras ofrece valores estadística-

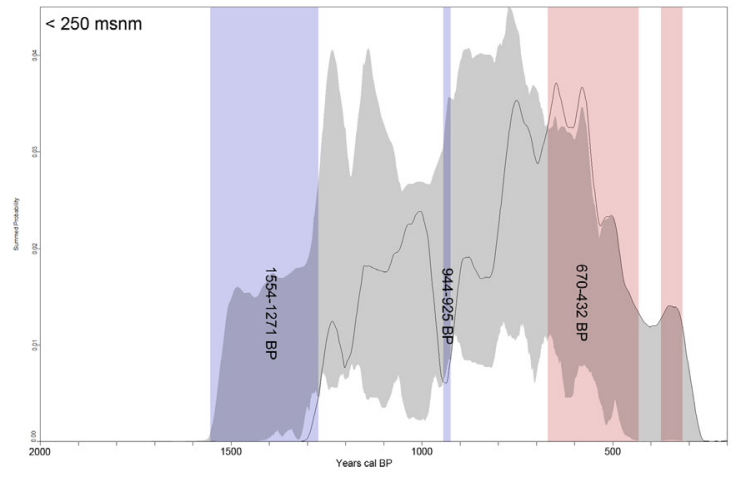

mente significativos como se refleja en la Fig. 8.

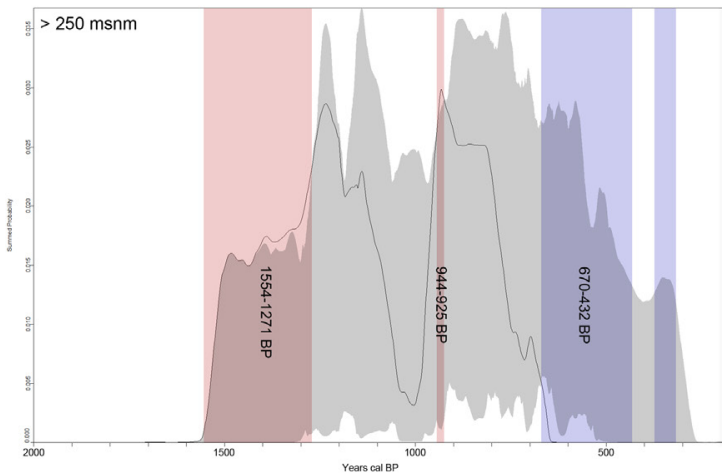

Figura 8: Distribución de suma de probabilidad por zonas. Espacios hasta los $250 \mathrm{msnm}$ : Número de dataciones: 94; número de bins: $31 ; \mathrm{p}=0,01099$. Desviación positiva significativa: $670-432$ BP y 373 317 BP; desviación negativa significativa: 1554-1271 BP y 944-925 BP .Espacios por encima de los 250 msnm: Número de dataciones: 77 ; número de bins: 20; $\mathrm{p}=0.01099$. Desviación positiva significativa: 1554-1271 BP y 944-925 BP; desviación negativa significativa: 670-432 BP y 373-317 BP. 


\section{Discusión}

Los resultados muestran una dinámica demográfica/social que está en sintonía con otros datos arqueológicos y con los procesos históricos que se han comenzado a proponer a partir del análisis cronocultural de las prácticas funerarias. Entender el proceso de poblamiento insular en una escala temporal amplia y secuenciada como la que aquí se propone permite acceder a comportamientos que hasta el momento no contaban con referencias claras para su encaje histórico.

\subsection{Los inicios del poblamiento efectivo de Gran Canaria}

Con las dataciones disponibles, así como con el cálculo de probabilidad presentado, el inicio del poblamiento de Gran Canaria no se retrotrae más allá del siglo III d.C. Sin embargo, es probable que la arribada de los primeros colonos a la isla se hubiera producido un poco antes, sin poder precisar el momento concreto. En este sentido, son varios los autores que advierten sobre el sesgo que puede introducir la pérdida de los contextos más antiguos, es decir un incremento de la posibilidad de desaparición de los yacimientos o secuencias arqueológicas de mayor antigüedad, por lo que $a$ priori quedarían subrepresentados en un muestreo global como este (Palmisano et al., 2017; Surovell et al., 2009). Podría añadirse que las actividades domésticas posiblemente generarían más cantidad de restos datables que los asociados a una práctica funeraria normalizada como la que evidencian los comportamientos identificados en los yacimientos aquí seleccionados (Prates et al., 2020). Por esta razón, y en este caso concreto, las estimaciones realizadas sobre fechas de restos humanos quizá suponen un ligero rejuvenecimiento del resultado obtenido.

El proceso colonizador de un nuevo territorio implica un tiempo variable de baja densidad de población y, por tanto, un lapso temporal con niveles reducidos de preservación / visibilidad del registro arqueológico (Rainbird, 2007; Prates et al., 2020). Más aún en un territorio insular como el de Gran Canaria en el que los primeros colonos tuvieron que reproducir y adaptar las estrategias económicas productoras traídas desde sus lugares de origen a un nuevo contexto geográfico. A ello se suma un contingente poblacional que en sus inicios no debió de ser muy cuantioso, tanto si estas poblaciones llegan empleando medios propios, como si son traídos a este territorio por terceros.

En consecuencia, es razonable pensar que la posibilidad de documentar registros arqueológicos de los primeros momentos de ocupación es más complicada que la de registrar aquellos situados algún tiempo después de la arribada inicial. La cuestión es cuánto tiempo es necesario para que esto suceda. En el caso de Gran Canaria es una pregunta que por ahora está por dilucidar, sobre todo porque hasta muy recientemente la huella arqueológica del primer poblamiento no ha sido objeto de un proceso de investigación particular. Tan solo se cuenta con un muestreo aleatorio que contempla las dataciones obtenidas en el marco de distintos proyectos y trabajos de investigación que no buscaban dar respuesta a esta cuestión.

El procedimiento ensayado retrasa en unos dos siglos el extremo de la calibración de la fecha fiable más antigua para Gran Canaria (1616 \pm 28 BP; cal 412- 540 d.C.). Quizá sea un margen temporal razonable para abarcar los procesos de consolidación de la población a los que se hacía referencia, situando la fecha de llegada de población continental norteafricana en torno a fines del siglo II o los primeros momentos del III d.C. Se trata, como ya se ha dicho, de una fecha que guarda coherencia con las dataciones fiables disponibles para el resto del Archipiélago.

Algunos trabajos biogeográficos recientes (Nascimento et al., 2016; 2020; Ravazzi et al., 2020), a partir de información diversa entre la que se incluyen dataciones radiocarbónicas, proponen un poblamiento más antiguo del Archipiélago, situándolo entre el 2300 y el 1800 BP. En el caso concreto de Gran Canaria, se plantea la posibilidad de un poblamiento anterior al aquí considerado, a partir de la datación del $1919 \pm 24$ BP de una muestra de la Laguna de Valleseco. Según estos autores, la muestra se asocia a cambios en el régimen local de fuegos y a la presencia de polen de cereal (Nascimento et al., 2016; Ravazzi et al., 2020). Sin descartar otros posibles escenarios, este equipo defiende que entre el 26 y el 131 cal CE (IntlCal13) esos dos indicadores serían pruebas tangibles de una isla antropizada en esas fechas. No obstante, se trata de unas dataciones que desde el punto de vista arqueológico presentan serios problemas de precisión y fiabilidad (Wright, 2007; Micó, 2020). En primer lugar, provienen de un depósito cuya 
dinámica de formación es ajena a la actividad humana, lo que les resta fiabilidad de cara a la asociación entre datación y evento arqueológico o histórico. Este problema se incrementa, en segundo lugar, por cuanto las muestras que proporcionan esas fechas son agregados de sedimento con presencia de carbones, lo que también representa una merma considerable de su fiabilidad. Se estarían datando especies vegetales diversas, de vida larga o corta, de dispar naturaleza, correspondientes a distintas cronologías, etc., lo que genera un margen de incertidumbre difícilmente cuantificable $\mathrm{y}$, por ello, con limitadas posibilidades informativas para la materia que aquí se aborda. En cualquier caso, si la fecha citada se recalibra con la curva Intcal 20, el escenario cronológico cambia ligeramente, situándose entre el 29-207 cal d.C., acercando su extremo más reciente al más antiguo del cálculo de probabilidad presentado en nuestros resultados. Con todo, esto no resuelve los problemas de fiabilidad y precisión a los que se hace referencia, por lo que habrá que aguardar a nuevas dataciones arqueológicas para ajustar un evento histórico que por el momento se establece entre la segunda mitad del II y principios del III d.C.

\subsection{Dinámica demográfica}

La comparación de la SPD con los modelos nulos teóricos muestra valores significativos en las dos propuestas planteadas, en especial cuando se recurre a los modelos nulos normalizados. En la segunda parte de la secuencia contemplada (a partir del 1000 BP aproximadamente), tanto el modelos exponencial como el logístico definen episodios positivos y negativos estadísticamente significativos en episodios temporales muy semejantes. Por el contrario, en la primera parte de la secuencia, en torno al 1600-1000 BP (350-950 d.C.) el patrón definido por el modelo nulo incluye un perfil escalonado, en algún caso con descensos acusados que, sin embargo, no sobrepasan la envolvente de simulación, lo que obliga a ser cautos en su explicación (Timpson et al., 2014). Llama la atención que los resultados expuestos describen un perfil de gráfica equivalente a la presentada en un trabajo previo (Pardo y Barceló, 2020: 491), aunque ahora con mayor significación estadística global y sin mostrar la desviación positiva, en torno a 1200-1100 BP (750-850 d.C.) y negativa, 1400 BP (550 d.C.) identificada en aquel modelo.
Quizá, los criterios de selección de muestra y las agrupaciones temporales que hemos asumido han reducido la densidad de dataciones para los primeros siglos de ocupación de Gran Canaria, atenuando el perfil de la gráfica en este tramo (Crema y Bevan, 2020b). A su vez, probablemente se incrementa la precisión al utilizar para el análisis exclusivamente dataciones obtenidas con materiales de ciclo corto.

En todo caso, quizá lo más significativo en este sentido es que, esta primera parte de la secuencia no parece responder a un patrón único de dinámica poblacional y probablemente en su configuración estén interviniendo otros factores además del crecimiento progresivo de la población. A escala global se observa una sucesión de eventos de crecimiento seguidos por episodios de estabilidad, con periodos puntuales de tendencia decreciente. Desde el 1400 BP (550 d.C.) parece describirse un modelo definido por la sucesión de crecimientos y descensos poblacionales, que varía siempre dentro de los márgenes del modelo nulo, incluso en su mayor descenso en torno al $1000 \mathrm{BP}$ (950 d.C.). A partir de la segunda parte, este esquema de ascensos y descensos se acentúa describiendo ahora un incremento que sobrepasa significativamente los límites del modelo nulo, para luego iniciar un rápido descenso de la curva.

Entre aproximadamente el 1600 y $1250 \mathrm{BP}$ (350-700 d.C.) se observa un proceso paulatino de crecimiento que aparentemente coincidiría con la consolidación de la ocupación de Gran Canaria. Los resultados no reflejarían los episodios más antiguos de la ocupación de la isla, sino una fase en la que se ha consolidado un patrón de crecimiento constante que alcanzará sus máximos en torno a 1300-1200 BP (650-750 d.C.). Coincidiendo con este intervalo cronológico se ha descrito la expansión de la población por la isla con un sensible incremento de yacimientos funerarios y domésticos fechados para estos momentos, como consecuencia de una diversificación en la ocupación y explotación del territorio (Moreno y González, 2016, Velasco, 2018; Moreno, 2020). Esta concomitancia podría respaldar que las variaciones de la curva, en efecto, son resultado de dinámicas sociales particulares observables en esta secuencia histórica, más aún si a esta lectura se incorporan otros cambios registrados en este mismo marco temporal. Es el caso del aumento en los marcadores de violencia física (Delgado et al., 2020), así como novedades en 
el registro funerario probablemente conectadas con la llegada a la isla de población norteafricana (Alberto et al., 2020). A partir de entonces, se asiste a un paulatino descenso que se prolonga hasta el $1000 \mathrm{BP}$ (950 d.C.) cuando alcanza su punto máximo. Precisamente, para esta primera parte de la secuencia la información arqueológica de contextos domésticos es muy escasa, limitando la capacidad de incorporar más variables al análisis socio-cultural. Sin embargo, los datos apuntan a que se trata de un período de cambios, a veces convulsos, que aún están por definir históricamente, pero que desembocan en una progresiva disminución de la huella arqueológica, y parece que de la población insular, en torno al cambio de milenio.

Por su parte, para la fase que va entre el siglo X d.C. y el momento de la conquista castellana (fines del XV) se tiene mayor volumen de información. En esta segunda parte de la secuencia se observa un incremento y posterior descenso muy significativos desde el punto de vista estadístico con respecto a cualquiera de los modelos nulos propuestos. Así, se registra un aumento acusado a partir de aproximadamente del 850-800 BP (1050-1100 d.C.) que se prolonga prácticamente hasta el $625 \mathrm{BP}(1325$ d.C.). En este marco temporal, considerando otros datos arqueológicos, se aprecian cambios significativos en el patrón de ocupación del territorio con respecto al modelo previo, el surgimiento de nuevos asentamientos, una sensi- ble intensificación de la actividad agrícola y de la explotación de los recursos marinos, el incremento de las huellas de violencia física, pero también de la violencia simbólica, acentuación de la asimetría social, así como la incorporación al registro arqueológico insular de ciertos elementos materiales que aparentemente no estaban presentes en momentos anteriores (Delgado, 2009; Morales, 2010; 2019; Rodríguez et al., 2012; del Pino et al., 2016; del Pino y Rodríguez, 2017; Henríquez et al., 2019; etc.). Además, la curva sugiere una dinámica social particular y acotada en el tiempo, en cuya configuración podría sumarse un nuevo episodio de llegada de población portadora de novedades culturales, entre las que se incluyen ciertas fórmulas sepulcrales (cistas y fosas; Fig. 9) (Alberto et al., 2020). Una serie de cambios en los que el incremento de habitantes en la isla también tendría un papel destacado en su explicación histórica. Si bien, y a juzgar por el patrón de la curva, no tanto como un agente causal de las novedades observables en el registro arqueológico, sino como el resultado de una serie de cambios acumulados que alcanza su máxima expresión en torno al 700 B.P. (1250 d.C.). A partir de este momento, se asiste a un descenso en la curva, que muestra valores negativos con respecto al modelo nulo en torno al 500 B.P. (1450 d.C.), muy probablemente en directa relación con la frecuentación de europeos en el mar de las islas y el proceso de conquista del archipiélago.

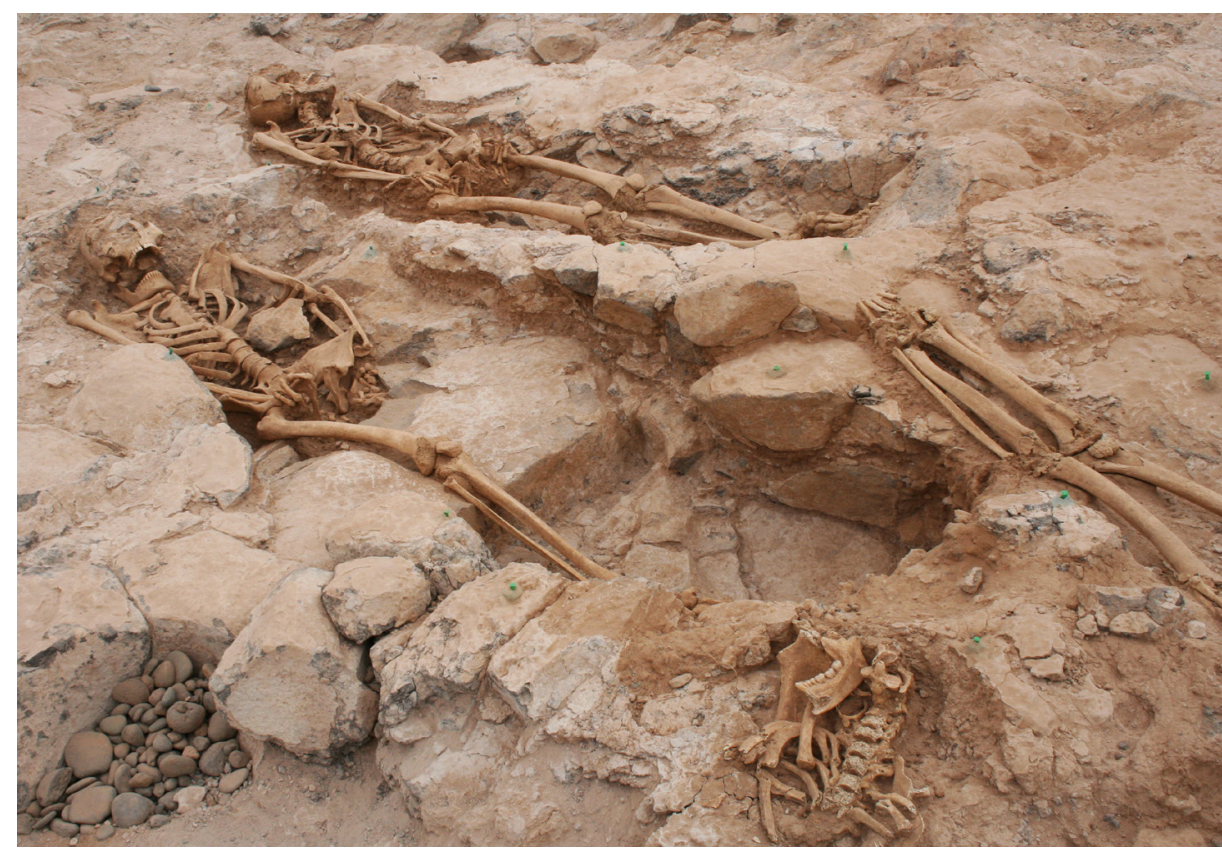

Figura 9: Necrópolis de fosas. Cementerio de Juan Primo (Gáldar). 
Los factores que intervienen en la configuración de esta dinámica poblacional son diversos y variados a lo largo de la secuencia histórica contemplada. Entre otros aspectos, una mirada territorial de los datos analizados puede contribuir a precisar este desarrollo insular. Así, al comparar los yacimientos entre los 250 msnm y los que superan esta cota se observan dos procesos claramente diferenciables, con una buena significación estadística y que, a todas luces, refuerza la lectura propuesta para el modelo global (Crema y Devan, 2020b).

Las medianías y el interior de Gran Canaria están ocupados desde el principio del poblamiento insular, describiéndose un crecimiento positivo por encima del modelo nulo hasta aproximadamente el 1300 BP (650 d.C.), aunque quizás pueda prolongarse algo más esa tendencia hasta $1250 \mathrm{BP}$ (700 d.C.). A partir de este momento, el descenso en la gráfica es evidente, y solo se observa una recuperación puntual en torno al 1000 BP (950 d.C.), para volver a caer por debajo de lo esperado en el modelo nulo en torno al 670 BP (1280 d.C.).

La ocupación de la franja litoral de Gran Canaria sigue una dinámica opuesta a la anterior. Hay que aguardar hasta prácticamente el 1300-1250 BP (fines del siglo VII o principios del VIII d.C.) para constatar las primeras ocupaciones costeras, como así parecen respaldar, por su lado, las dataciones más antiguas de espacios domésticos asociadas a este entono (Martín, 2000; Rodríguez et al., 2012 y Velasco, 2018). Sin embargo, no es hasta el 1000-900 BP (finales del siglo X o a principios del XI) que la ocupación de la franja litoral se consolida, llegando a alcanzar desde 600 BP (mediados del siglo XIV) rangos que sobrepasan los límites del modelo nulo. A este respecto, la datación a priori más antigua del litoral procede de una concha marina del yacimiento arqueológico de la playa de Aguadulce (Martín, 2000), pero su calibración proporciona un margen de incertidumbre que sitúa la primera ocupación de este enclave costero entre los siglos V y VIII d.C. (1930 \pm 40 BP; 474-771 cal d.C.). Aplicando criterios de corrección (Parker et al., 2020) que en su día no fueron considerados, el rango de probabilidad de esta datación se amplía considerablemente: 331872 cal d.C. (http://calib.org/calib/calib.html), disminuyendo de manera notable su precisión de cara a abordar con mínimas garantías la cuestión que ahora se trata. Por su parte, los enclaves costeros fechados con muestras te- rrestres de ciclo corto, como por ejemplo el caso de Dunas de Maspalomas o la Playa de Sardina, parecen ajustarse a lo antes señalado, situándose su ocupación a partir de los siglos VII-VIII d.C. (Rodríguez et al. 2012). Por todo ello puede mantenerse que la importancia y frecuencia de los asentamientos próximos al mar se mantiene aparentemente baja en estos primeros siglos, al menos en comparación con lo que se constata en periodos posteriores.

Según estas circunstancias, puede plantearse que, entre otras cuestiones, las variaciones en la gráfica de la SPD están reflejando cambios en la dinámica social que tienen una proyección global y, en particular, en el régimen económico insular. Si valoramos en su conjunto los resultados expuestos, el resto de información arqueológica disponible y los estudios territoriales, se observa un cambio evidente. En concreto, variando desde un modelo de producción de corte agropastoril, en el que la ganadería tendría un peso considerable, dominante en la primera parte de la secuencia, a otro en el que el protagonismo pasa a la producción cerealista y la explotación de los recursos marinos, propio de la última fase del poblamiento. Este cambio explicaría, por un lado las tendencias observadas en la ocupación del territorio y, por otro, las desigualdades en el régimen demográfico de uno y otro momento. De tal modo que el régimen agrícola intensivo que se ha descrito para Gran Canaria a partir de los inicios del segundo milenio, habría favorecido un importante incremento poblacional en los últimos siglos de ocupación de la isla. Desde esta perspectiva, el régimen demográfico de los antiguos canarios que describen las fuentes narrativas europeas no sería el resultado de un crecimiento paulatino cuyos orígenes habría que buscar en los inicios del poblamiento, sino la consecuencia de una dinámica histórica particular de las últimas centurias y, en especial, a partir de la segunda mitad del XIII d.C. Por otra parte, para los primeros siglos todo apunta a un patrón de menor carga demográfica a escala insular, con incrementos pausados que son seguidos por episodios de estabilidad e incluso decrecimiento más o menos acentuado.

Desde el punto de vista territorial, los datos sugieren un progresivo desplazamiento de la ocupación humana desde el interior y las medianías hacia la franja litoral. Este proceso, en sintonía con los cambios económicos, se hace patente a partir del siglo XI d.C. y alcanza su máximo en la segunda mitad del XIII. El per- 
fil de la gráfica en el período que va del siglo XII al XV más que en un modelo logístico de crecimiento poblacional, encaja mejor en uno de auge (boom) y caída (Brown et al., 2019), lo que resulta coherente con otras evidencias arqueológicas (véase, por ejemplo, Lécuyer et $a l ., 2021)$. Una situación de cambio que puede relacionarse cronológicamente con la aparición de novedades en el registro arqueológico insular (por ejemplo, tipología de asentamientos y nuevas fórmulas funerarias), con testimonios de una intensificación económica cerealista y del aprovechamiento del medio marino, auge de los espacios de almacenamiento fortificados, huellas de especialización laboral y de jerarquización social, etc. La SPD en este tramo cronológico es un exponente más de un período de importantes transformaciones en Gran Canaria que dada su trascendencia y la ausencia de precedentes en el registro material insular, parece que pudo ser dinamizado o co-dinamizado por la llegada de nueva población norteafricana a la isla en torno a finales del siglo X o principios del XI d.C.

\section{Conclusiones}

Desde una perspectiva global, los datos de distribución del sumatorio de probabilidad de las dataciones revelan una tendencia a largo plazo que tiene encaje en una curva logística, pero en la que se registran momentos de crecimiento y decrecimiento puntuales indicadoras de dinámicas particulares que responden a coyunturas históricas concretas. No puede hablarse por ello, de una dinámica poblacional de crecimiento uniforme o exponencial en la secuencia histórica contemplada. En este sentido, quizá el aspecto más destacado de la lectura de la gráfica de la SPD es la expresión de dos episodios que ayudan a caracterizar la secuencia histórica de los antiguos canarios. El primer episodio podría situarse en torno a 1350-1200 BP (600-750 d.C.) y, como ya se ha dicho en otros trabajos, coincide con la incorporación al registro arqueológico insular de las grandes necrópolis tumulares (Fig. 10) y, con ellas, cambios aparentes en la ordenación del territorio y en la ordenación de las relaciones sociales. Las huellas de la ocupación humana se diversifican, acaso en un marco de cierta inestabilidad y conflictividad que explicaría la alta concentración de evidencias de violencia física letal en estos momentos. La concurrencia de un evento migratorio de nueva población continental también se apunta como agente causal para algunas de estas innovaciones, y los procesos económicos y sociales que se dan a partir de estos momentos.

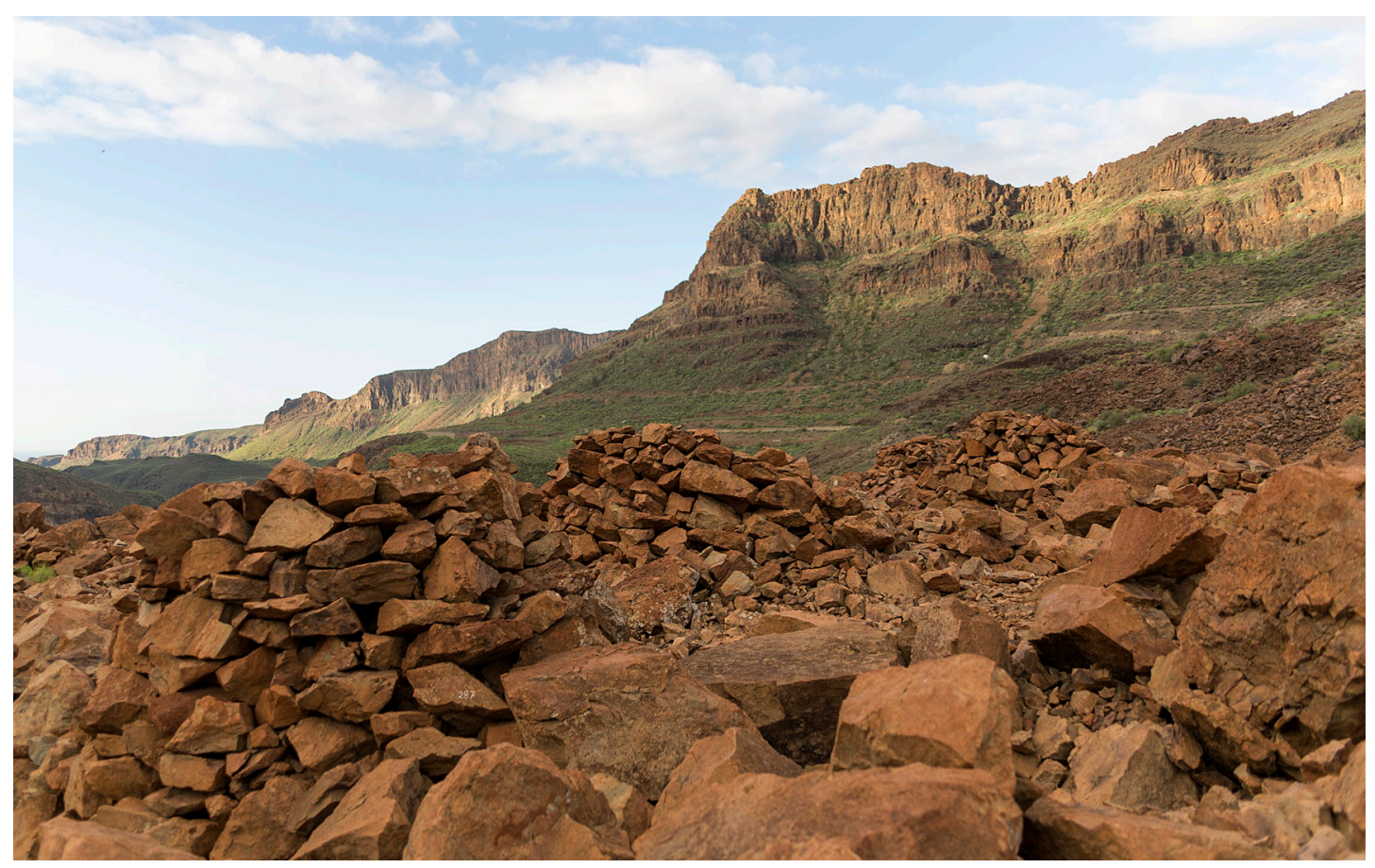

Figura 10: Necrópolis tumular de Arteara (San Bartolomé de Tirajana) 
En todo caso, llama la atención que tras un proceso ascendente que arrancaría en los primeros momentos del poblamiento, este primer episodio se estabiliza temporalmente, para iniciar un declive en torno al 1150 BP (800 d.C.) hasta aproximadamente el $1000 \mathrm{BP}$ (950 d.C.), donde alcanza unos mínimos que no van a repetirse hasta la conquista europea. Es cierto que deben adoptarse cautelas en la valoración de estas circunstancias, pues en ningún caso se sobrepasan los límites que marcan los modelos nulos, pero es evidente que se describe una tendencia general que insinúa un escenario de pérdida de población.

El segundo episodio, a partir del X-XI d. C. es mucho más significativo desde el punto de vista estadístico y se concreta en un perfil de curva indicativo de una dinámica de crecimiento ininterrumpido que alcanza su máximo en torno a mediados del siglo XIII. Aparentemente, este crecimiento es tan rápido que, en un modelo teórico poblacional, pudiera interpretarse como un proceso que conduce a un desequilibrio entre población y recursos (incluido el modo en el que se distribuyen), lo que trae aparejado un descenso demográfico posterior. Una dinámica coherente con la información que proporcionan otros marcadores arqueológicos, en particular los que tienen que ver con la intensificación de la producción agrícola, la custodia de recursos alimenticios en espacios de fácil defensa, la intensificación de la pesca, los patrones de dieta, etc.

Desde el punto de vista territorial, además del asentamiento inicial, los dos grandes periodos de la secuencia tienen su correspondencia con cambios en el paisaje humano de Gran Canaria. Mientras que en los primeros siglos de ocupación los asentamientos se concentran preferentemente por encima de los $250 \mathrm{msnm}$, los cambios que se producen en la isla a partir del X-XI d.C. conllevan que las principales densidades de población se sitúen en la franja litoral. Las medianías y el interior de Gran Canaria no se abandonan, pero el modelo global indica que los máximos demográficos se concentran en las grandes vegas agrícolas y cercanas a una costa que también brinda importantes recursos alimenticios a través de la pesca.

El sumatorio de la distribución de probabilidades de dataciones, como ha tratado de exponerse, es una herramienta idónea para el planteamiento de modelos generales a largo plazo en la dinámica poblacional de los antiguos canarios. Aun tratándose de una aproximación provisional que se irá matizando con la aportación de nuevas dataciones, proporciona un marco de referencia coherente desde el que explicar los fenómenos arqueológicos desvelados en los últimos años.

\section{Bibliografía}

Alberto Barroso, V.; Delgado Darias, T.; Moreno Benítez, M. y Velasco Vázquez, J. (2019): La dimensión temporal y el fenómeno sepulcral entre los antiguos canarios. Zephyrus, LXXXIV: 139-160. https://doi. org/10.14201/zephyrus201984139160

Alberto Barroso, V.; Velasco Vázquez, J.; Delgado Darias, T. y Moreno Benítez, M. (2020): Los antiguos canarios ante la muerte. Tradición vs ruptura. Gran Canaria las Huellas del Tiempo (J. Afonso, ed.), Instituto de Estudios Hispánicos, Tenerife: 13-40.

Bernabeu Aubán, J.; Jiménez Puerto, J.; Escribá Ruiz, P. y Pardo Gordó, S. (2018): C14 y poblamiento en las comarcas centro-meridionales del País Valenciano (c. 7000-1500 cal BC). Recerques del Museu d'Alcoi, 27: 35-48.

Blakeslee, D.J. (1994): Reassessment of some radiocarbon dates from the Central Plains. Plains Anthropologist, 39(148): 103-210. https://doi.org/10.1080/2052546.1994.11931727

Brown, A. y Crema, E.R. (2019): Māori Population Growth in Pre-contact New Zealand: Regional Population Dynamics Inferred From Summed Probability Distributions of Radiocarbon Dates. The Journal of Island and Coastal Archaeology: 1-19. https://doi.org/10.1080/15564894.2019.1605429

Carleton, W.C. y Groucutt, H.S. (2020): Sum things are not what they seem: Problems with point-wise interpretations and quantitative analyses of proxies based on aggregated radiocarbon dates. The Holocene, 0959683620981700. https://doi.org/10.1177/0959683620981700.

Contreras, D. y Meadows, J. (2020): Estudios paleodemográficos basados en conjuntos de dataciones radiométricas. Una revision crítica. Métodos cronométricos en arqueología, historia y paleontología (J. Barceló y B. Morell, eds.), Dextra, Barcelona: 497-520. 
Crema, E.R., Bevan, A. y Shennan, S. (2017): Spatio-temporal approaches to archaeological radiocarbon dates. Journal of Archaeological Science, 87: 1-9. https://doi.org/10.1016/j.jas.2017.09.007.

Crema, E.R. y Bevan, A. (2020a): Analysing radiocarbon dates using the rcarbon package. [URL: https:// cran.r-project.org/web/packages/rcarbon/vignettes/rcarbon.html]. [Último acceso: 29/01/2021].

Crema, E.R. y Bevan, A. (2020b): Inference from Large Sets of Radiocarbon Dates: Software and Methods. Radiocarbon: 1-17. https://doi.org/10.1017/rdc.2020.95.

Crema, E.R. y Kobayashi, K. (2020): A multi-proxy inference of Jōmon population dynamics using bayesian phase models, residential data, and summed probability distribution of $14 \mathrm{C}$ dates. Journal of Archaeological Science, 117: 105136. https://doi.org/10.1016/j.jas.2020.105136

del Pino Curbelo, M.; Rodríguez Rodríguez, A.; Buxeda i Garrigós, J.; Mangas Viñuela, J.; Day, P.; González Quintero, P. y Moreno Benítez, M. (2016): Las cerámicas aborígenes de Gran Canaria (Islas Canarias) a través del yacimiento de La Cerera: materias primas, tecnología y función. Trabajos de Prehistoria, 73(1): 90-114. https://doi.org/10.3989/tp.2016.12165

del Pino, M. y Rodríguez, A. (2017): Propuesta para la clasificación de los materiales cerámicos de tradición aborigen de la isla de Gran Canaria (Islas Canarias). Lucentum, 36: 9-31.

Delgado, T. (2009): La historia en los dientes: una aproximación a la prehistoria de Gran Canaria desde la antropología dental. Ediciones del Cabildo de Gran Canaria. Las Palmas de Gran Canaria.

Delgado, T. Velasco, J. y Alberto, V. (2020). Isolation and violence on an oceanic island: lethal injuries in a Pre-Hispanic burial in Gran Canaria (Canary Islands, Spain). The Journal of Island and Coastal Archaeology, 1-19. DOI: 10.1080/15564894.2020.1783036

DiNapoli, R.J.; Rieth, T.M.; Lipo, C.P. y Hunt, T.L. (2020): A model-based approach to the tempo of "collapse": The case of Rapa Nui (Easter Island). Journal of Archaeological Science, 105094. https://doi. org/10.1016/j.jas.2020.105094

Henríquez, P.; Morales, J.; Vidal, P.; Santana, J. y Rodríguez, A. (2019): Arqueoentomología y arqueobotánica de los espacios de almacenamiento a largo plazo: el granero de Risco Pintado, Temisas (Gran Canaria). Trabajos de Prehistoria, 76(1): 120-137.

Hinz, M. (2020): Sensitivity of Radiocarbon Sum Calibration. Journal of Computer Applications in Archaeology, 3(1). http://doi.org/10.5334/jcaa.53

Jiménez González, J. (1999): Gran Canaria prehispánica. Un modelo desde la arqueología antropológica. Centro de la Cultura Popular Canaria, Santa Cruz de Tenerife.

Lécuyer, C.; Goedert, J.; Klee, J.; Clauzel, T.; Richardin, P.; Fourel, F.;Delgado-Darias, T; Alberto-Barroso, V.; Velasco-Vázquez, J., Betancort, J.F.; Amiot, R., Maréchal, C. y Flandrois, J. P. (2021): Climatic change and diet of the pre-Hispanic population of Gran Canaria (Canary Archipelago, Spain) during the Medieval Warm Period and Little Ice Age. Journal of Archaeological Science, 128, 105336. https://doi. org/10.1016/j.jas.2021.105336.

Martín Rodríguez, E. (2000): Dataciones absolutas para los yacimientos de Risco Chimirique (Tejeda) y Playa de Aguadulce (Telde). Vegueta, 5: 29-46

Mederos Martín, A. (2019): Un enfrentamiento desigual: Baja demografía y difícil resistencia en la conquista de las Islas Canarias. Anuario de Estudios Atlánticos, 65: 1-32.

Micó, R. (2020): El muestreo para la datación por radiocarbono: consideraciones y criterios arqueológicos. Métodos cronométricos en arqueología, historia y paleontología (J. Barceló y B. Morell, eds.), Dextra, Barcelona: 275-295.

Morales, J. (2010): El uso de las plantas en la prehistoria de Gran Canaria: alimentación, agricultura y ecología. Ediciones Cabildo de Gran Canaria.

Morales, J. (2019): Los guardianes de las semillas. Origen y evolución de la agricultura en Gran Canaria. Col. La isla de los canarios. Ediciones Cabildo de Gran Canaria, Las Palmas de Gran Canaria.

Moreno Benítez, M. y González Quintero, P. (2016): Una perspectiva territorial al uso del suelo en la Gran Canaria prehispánica (siglos XI-XV). Tabona, 20: 9-32.

Moreno, M. (2020): El tiempo perdido. Un relato arqueológico del la Tirajana indígena. Tibicena Publicaciones, Las Palmas de Gran Canaria.

Mulrooney, M.A. (2013): An island-wide assessment of the chronology of settlement and land use on Rapa Nui (Easter Island) based on radiocarbon data. Journal of Archaeological Science, 40(12): 4377-4399. https://doi.org/10.1016/j.jas.2013.06.020. 
Nascimento, L.; Nogué, S.; Criado, C.; Ravazzi, C.; Whittaker, R.J.; Willis, K.J. y Fernández-Palacios, J.M. (2016): Reconstructing Holocene vegetation on the island of Gran Canaria before and after human colonization. The Holocene, 26(1): 113-125. https://doi.org/10.1177/0959683615596836.

Nascimento, L.; Nogué, S.; Naranjo-Cigala, A.; Criado, C.; McGlone, M.; Fernández-Palacios, E. y Fernández-Palacios, J.M. (2020): Human impact and ecological changes during prehistoric settlement on the Canary Islands. Quaternary Science Reviews, 239: 106332. https://doi.org/10.1016/j.quascirev.2020.106332.

Onrubia Pintado, J. (2003). La isla de los Guanartemes. Territorio, sociedad y poder en la Gran Canaria indigena (siglos XIV-XV). Ediciones del Cabildo de Gran Canaria, Las Palmas de Gran Canaria.

Palmisano, A.; Bevan, A. y Shennan, S. (2017): Comparing archaeological proxies for long-term population patterns: An example from central Italy. Journal of Archaeological Science, 87: 59-72. https://doi. org/10.1016/j.jas.2017.10.001.

Palmisano, A.; Lawrence, D.; de Gruchy, M.W.; Bevan, A. y Shennan, S. (2020): Holocene regional population dynamics and climatic trends in the Near East: A first comparison using archaeo-demographic proxies. Quaternary Science Reviews, 252, 106739. https://doi.org/10.1016/j.quascirev.2020.106739.

Pardo-Gordó, S. y Barceló, J. (2020): Paleodemografía y dataciones radiométricas en la península ibérica. Métodos cronométricos en arqueología, historia y paleontología (J. Barceló y B. Morell, eds.), Dextra, Barcelona: 477-495.

Parker, W., Yanes, Y., Mesa, E., Hernández, J. C., Pais, J., Soto, N. y Surge, D. (2020): Shellfish exploitation in the western Canary Islands over the last two millennia. Environmental Archaeology, 25(1): 14-36.

Porčić, M., Blagojević, T., Pendić, J., \& Stefanović, S. (2021): The Neolithic Demographic Transition in the Central Balkans: population dynamics reconstruction based on new radiocarbon evidence. Philosophical Transactions of the Royal Society B, 376(1816), 20190712. https://doi.org/10.1098/rstb.2019.0712.

Prates, L.; Politis, G. G. y Perez, S.I. (2020): Rapid radiation of humans in South America after the last glacial maximum: A radiocarbon-based study. PloS one, 15(7), e0236023. https://doi.org/10.1371/journal. pone. 0236023.

Puleston, C. y Winterhalder, B. (2019): Demography, environment, and human behavior. Handbook of Evolutionary Research in Archaeology, (A.M. Prentis, ed.), Springer, Cham: 311-335.

Quintus, S.; Huebert, J.; Day, S.; Lincoln, N.; Yoo, K.; Lee, T.; Moleala, F. y \& Autufuga, D. (2020): Tempo and trajectory of the built landscape on Ta ' $u$ island, Manu 'a group, American Samoa: integrating extensive radiocarbon dating with joint posterior modeling. Radiocarbon, 62(5): 1317-1337. https://doi. org/10.1017/RDC.2020.60

Rainbird, P. (2007): The archaeology of islands. Cambridge University Press, Cambridge.

Ramsey, C.B. (2009): Dealing with outliers and offsets in radiocarbon dating. Radiocarbon, 51(3): 10231045. https://doi.org/10.1017/S0033822200034093.

Ravazzi, C.; Mariani, M.; Criado, C.; Garozzo, L.; Naranjo-Cigala, A.; Perez-Torrado, F. J.;... y de Nascimento, L. (2020): The influence of natural fire and cultural practices on island ecosystems: Insights from a 4,800 year record from Gran Canaria, Canary Islands. Journal of Biogeography. https://doi. org/10.1111/jbi.13995.

R Core Team. 2020. R: A language and environment for statistical computing. R Foundation for Statistical Computing. Vienna, Austria. URL http://www.R-project.org.

Reimer, P.J.; Austin, W.; Bard, E.; Bayliss, A.; Blackwell, P.G.; Ramsey, C.B.; Butzin, M.; Cheng, H.; Edwards, R.L.; Friedrich, M.; Grootes, P.M.; Guilderson, T.P.; Hajdas, I.; Heaton, T.J.; Hogg, A.G.: Hughen, K.A.: Kromer, B.; Manning, S.W.; Muscheler, R.; Palmer, J.G.; Pearson, C.; Plicht, J. van der; Reimer, R.W.; Richards, D.A.; Scott, E.M.; Southon, J.R.; Turney, C.S.; Wacker, L.; Adolphi, F.; Büntgen, U.; Capano, M.; Fahrni, S.M.; Fogtmann-Schulz, A.; Friedrich, R.; Köhler, P.; Kudsk, S.; Miyake, F.; Olsen, J.; Reinig, F.; Sakamoto, M.; Sookdeo, A. y Talamo, S. (2020): The IntCal Northern Hemisphere Radiocarbon Age Calibration Curve (0-55 Cal kBP). Radiocarbon: 1-33. https://doi.org/10.1017/ RDC.2020.41.

Rodríguez Rodríguez, A.; Morales, J.; Pino Curbelo, M.; Naranjo-Mayor, Y.; Martín Rodríguez, E. y González Marrero, M.C. (2012): Espacios de producción especializada, excedentes y estratificación social en la Gran Canaria pre-europea. Tabona, 19: 101-123.

Roscoe, P.; Sandweiss, D.H. y Robinson, E. (2021): Population density and size facilitate interactive capacity and the rise of the state. Philosophical Transactions of the Royal Society B, 376(1816), 20190725. https://doi.org/10.1098/rstb.2019.0725. 
Shennan, S.; Downey, S.; Timpson., A; Edinborough, K; Colledge, S.; Kerig, T.; Manning, K. y Thomas, M. (2013): Regional population collapse followed initial agriculture booms in mid-Holocene Europe. Nature Communications 4: ncomms3486. https://doi.org/10.1038/ncomms3486.

Surovell, T.A.; Byrd Finley, J.; Smith, G.M.; Brantingham, P.J. y Kelly, R. (2009): Correcting temporal frequency distributions for taphonomic bias. Journal of Archaeological Science, 36: 1715-1724. https:// doi.org/10.1016/j.jas.2009.03.029.

Timpson, A.; Colledge, S.; Crema, E.; Edinborough, K.; Kerig, T.; Manning, K.; Thomas. M.G. y Shennan, S. (2014): Reconstructing regional population fluctuations in the European Neolithic using radiocarbon dates: a new case-study using an improved method. Journal of Archaeological Science, 52: 549-57. https://doi.org/10.1016/j.jas.2014.08.011.

Velasco-Vázquez, J. (2018): La isla de los canarios. Gentes, tiempos y lugares. Ediciones del Cabildo de Gran Canaria, Las Palmas de Gran Canaria.

Velasco-Vázquez, J.; Alberto-Barroso, V.; Delgado Darías, T.; Moreno-Benítez, M.M.; Lécuyer, C. y Richardin, P. (2020): Poblamiento, colonización y primera historia de Canarias: El C14 como paradigma. Anuario de Estudios Atlánticos, 66: 1-24.

Weninger, B.; Clare, L.; Jöris, O.; Jung, R. y Edinborough, K. (2015): Quantum theory of radiocarbon calibration. World Archaeology 47: 543-66. https://doi.org/10.1080/00438243.2015.1064022.

Williams, A.N. (2012): The use of summed radiocarbon probability distributions in archaeology: a review of methods. Journal of Archaeological Science, 39(3): 578-589. https://doi.org/10.1016/j.jas.2011.07.014.

Wright, D. (2017): Accuracy vs precision: Understanding potential errors from radiocarbon dating on African landscapes. African Archaeological Review, 34: 303-319. https://doi.org/10.1007/s10437-0179257-z

\section{Anexo. Tabla 1}

\begin{tabular}{|l|l|l|r|r|r|r|}
\hline \multicolumn{1}{|c|}{ Nombre } & \multicolumn{1}{|c|}{ Muestra } & \multicolumn{1}{c|}{$\begin{array}{c}\text { Código } \\
\text { laboratorio }\end{array}$} & $\begin{array}{c}\text { Edad } \\
\text { convencional BP }\end{array}$ & DE & Tipo & Territorio \\
\hline Acarreaderos & Hueso humano & Beta 361285 & 790 & 30 & 1 & $\mathbf{1}$ \\
\hline Acarreaderos & Hueso humano & Beta 370948 & 280 & 30 & 1 & $\mathbf{1}$ \\
\hline Acusa & Piel momia & GRO 1188 & 1380 & 60 & 1 & 2 \\
\hline Acusa & Tejido vegetal & Beta 468994 & 1310 & 30 & 1 & 2 \\
\hline Acusa & Piel fardo & Beta 391058 & 1270 & 30 & 1 & 2 \\
\hline Acusa & Hueso humano & SacA500016 & 1310 & 30 & 1 & 2 \\
\hline Acusa & Piel humana & Beta-468988 & 1300 & 30 & 1 & 2 \\
\hline Acusa & Hueso humano & SacA500017 & 1275 & 30 & 1 & 2 \\
\hline Acusa & Junco mortaja & Beta 539739 & 1230 & 30 & 1 & 2 \\
\hline Acusa & Tejido blando & Beta 565457 & 1260 & 30 & 1 & 2 \\
\hline Acusa & Tejido blando & Beta 565458 & 1610 & 30 & 1 & 2 \\
\hline Acusa & Tejido blando & Beta 510717 & 1540 & 30 & 1 & 2 \\
\hline Acusa & Tejido blando & Beta 510718 & 630 & 40 & 3 & 2 \\
\hline Agujero & Hueso humano & Beta 261235 & 610 & 40 & 3 & 1 \\
\hline Agujero & Hueso humano & Beta 261236 & 640 & 40 & 3 & 1 \\
\hline Agujero & Hueso humano & Beta 261237 & 530 & 40 & 3 & 1 \\
\hline Agujero & Hueso humano & Beta 261238 & 690 & 40 & 3 & 1 \\
\hline Agujero & Hueso humano & Beta 261239 & 770 & 40 & 3 & 1 \\
\hline Agujero & Hueso humano & Beta 261240 & 40 & 3 & 1 \\
\hline Agujero & Hueso humano & Beta 261241 & & & 1 \\
\hline
\end{tabular}




\begin{tabular}{|c|c|c|c|c|c|c|}
\hline Agujero & Hueso humano & SacA50002 & 635 & 30 & 3 & 1 \\
\hline Agujero & Hueso humano & SacA50003 & 555 & 30 & 3 & 1 \\
\hline Agujero & Hueso humano & SacA50004 & 680 & 30 & 3 & 1 \\
\hline Agujero & Hueso humano & SacA50005 & 585 & 30 & 3 & 1 \\
\hline Agujero & Hueso humano & SacA50007 & 655 & 30 & 3 & 1 \\
\hline Agujero & Hueso humano & Beta 565463 & 480 & 30 & 3 & 1 \\
\hline Angostura & Hueso humano & Beta 539747 & 1460 & 30 & 1 & 2 \\
\hline Angostura & Hueso humano & Beta 539748 & 1500 & 30 & 1 & 2 \\
\hline Angostura & Hueso humano & Beta 539745 & 1590 & 30 & 1 & 2 \\
\hline Angostura & Hueso humano & Beta 539746 & 920 & 30 & 1 & 2 \\
\hline Arteara & Junco mortaja & Beta 346111 & 970 & 30 & 2 & 2 \\
\hline Arteara & Junco mortaja & Beta 349001 & 1220 & 30 & 2 & 2 \\
\hline Arteara & Hueso humano & Beta 565472 & 1050 & 30 & 2 & 2 \\
\hline Arteara & Hueso humano & Beta 565473 & 900 & 30 & 2 & 2 \\
\hline Puerca & Hueso humano & DAMS 015878 & 873 & 35 & 1 & 1 \\
\hline Puerca & Hueso animal & DAMS 015879 & 624 & 25 & 1 & 1 \\
\hline Bco. Hondo & Hueso humano & Beta 539728 & 830 & 30 & 1 & 1 \\
\hline Bco. Hondo & Hueso humano & Beta 539729 & 830 & 30 & 1 & 1 \\
\hline Bco. Hondo & Hueso humano & Beta 539730 & 900 & 30 & 1 & 1 \\
\hline Bentayga & Hueso humano & Beta & 1040 & 30 & 1 & 2 \\
\hline Bentayga & Hueso humano & Beta 565466 & 768 & 25 & 1 & 2 \\
\hline Cardones & Hueso humano & Beta 539725 & 450 & 30 & 1 & 1 \\
\hline Cardones & Hueso humano & Beta 539726 & 1150 & 30 & 1 & 1 \\
\hline Linagua & Hueso humano & Beta 535735 & 890 & 30 & 1 & 2 \\
\hline Linagua & Hueso humano & Beta 535741 & 920 & 30 & 1 & 2 \\
\hline Cendro & Hueso humano & Beta 539743 & 970 & 30 & 3 & 1 \\
\hline Cendro & Hueso animal & Beta 565477 & 850 & 30 & 3 & 1 \\
\hline Cendro & Hueso humano & Beta 565478 & 900 & 30 & 3 & 1 \\
\hline Cendro & Hueso humano & Beta 561333 & 850 & 30 & 3 & 1 \\
\hline Cendro & Hueso humano & Beta 561334 & 860 & 30 & 3 & 1 \\
\hline Cendro & Hueso humano & Beta 561335 & 860 & 30 & 3 & 1 \\
\hline Chimirique & Junco mortaja & Beta 131031 & 890 & 50 & 1 & 2 \\
\hline Crucecitas & Hueso humano & Beta 539732 & 750 & 30 & 3 & 1 \\
\hline Crucecitas & Hueso humano & Beta 302330 & 800 & 30 & 1 & 1 \\
\hline Cabezo & Hueso humano & Beta 221674 & 1170 & 40 & 1 & 1 \\
\hline Cabezo & Hueso humano & Beta 221673 & 810 & 40 & 1 & 1 \\
\hline Drago & Hueso humano & Beta 539731 & 870 & 30 & 1 & 1 \\
\hline Drago & Hueso humano & Beta 561337 & 830 & 30 & 1 & 1 \\
\hline Drago & Hueso humano & Beta 561338 & 1590 & 30 & 1 & 1 \\
\hline Drago & Hueso humano & Beta 561339 & 790 & 30 & 1 & 1 \\
\hline Drago & Hueso humano & Beta 561340 & 850 & 30 & 1 & 1 \\
\hline Drago & Hueso humano & Beta 561341 & 1600 & 30 & 1 & 1 \\
\hline Drago & Hueso animal & Beta 561342 & 700 & 30 & 1 & 1 \\
\hline
\end{tabular}




\begin{tabular}{|c|c|c|c|c|c|c|}
\hline Risco & Hueso humano & Beta 302335 & 690 & 30 & 3 & 1 \\
\hline Risco & Hueso humano & Beta 302336 & 630 & 30 & 3 & 1 \\
\hline Pajito & Hueso humano & Beta 510708 & 890 & 30 & 1 & 2 \\
\hline Espigon & Hueso humano & DAMS 032110 & 1485 & 29 & 1 & 2 \\
\hline Fortaleza & Hueso humano & $\begin{array}{l}\text { DAMS } 021461 \\
977\end{array}$ & 1496 & 33 & 1 & 2 \\
\hline Fortaleza & Hueso humano & $\begin{array}{l}\text { DAMS } 021462 \\
1837\end{array}$ & 1469 & 25 & 1 & 2 \\
\hline Fortaleza & Hueso humano & DAMS 032109 & 1146 & 26 & 1 & 2 \\
\hline Fortaleza & Diente & DAMS 032108 & 1210 & 23 & 1 & 2 \\
\hline Fortaleza & Hueso humano & DAMS 032119 & 860 & 34 & 1 & 2 \\
\hline Fortaleza & Hueso humano & DAMS 032120 & 1071 & 29 & 1 & 2 \\
\hline Fortaleza & Hueso humano & DAMS 032122 & 1323 & 31 & 1 & 2 \\
\hline Guanarteme & Hueso humano & Beta 565467 & 1120 & 30 & 1 & 2 \\
\hline Guayadeque & Hueso humano & Beta 468985 & 1500 & 30 & 1 & 2 \\
\hline Guayadeque & Hueso humano & Beta 468986 & 1460 & 30 & 1 & 2 \\
\hline Guayadeque & Hueso humano & Beta 468987 & 1480 & 30 & 1 & 2 \\
\hline Guayadeque & Tejido blando & Beta 468992 & 1040 & 30 & 1 & 2 \\
\hline Guayadeque & Hueso animal & Beta 468995 & 1250 & 30 & 1 & 2 \\
\hline Guayadeque & Piel mortaja & GRO 1189 & 1410 & 60 & 1 & 2 \\
\hline Guayadeque & Hueso humano & 1A1066 & 1460 & 30 & 1 & 2 \\
\hline Guayadeque & Hueso humano & Beta 468991 & 1550 & 30 & 1 & 2 \\
\hline Guayadeque & Hueso humano & Beta 46890 & 1370 & 30 & 1 & 2 \\
\hline Guayadeque & Hueso humano & $1 \mathrm{~A} 1067$ & 1265 & 30 & 1 & 2 \\
\hline Guayadeque & Hueso humano & 1A1068 & 1315 & 30 & 1 & 2 \\
\hline Guayadeque & Hueso humano & SacA500021 & 1310 & 30 & 1 & 2 \\
\hline Guayadeque & Hueso humano & SacA500023 & 1325 & 30 & 1 & 2 \\
\hline Guayadeque & Hueso humano & SacA500024 & 1365 & 30 & 1 & 2 \\
\hline Guayadeque & Hueso humano & Beta 510716 & 1180 & 30 & 1 & 2 \\
\hline Gua_Arg & Hueso humano & Beta 391059 & 1570 & 30 & 1 & 2 \\
\hline Gua_Arg & Hueso humano & Beta 468989 & 1470 & 30 & 1 & 2 \\
\hline Guayadeque & Hueso humano & DAMS 027470 & 768 & 25 & 1 & 2 \\
\hline Guayadeque & Tejido blando & Beta 539327 & 1500 & 30 & 1 & 2 \\
\hline Guayadeque & Tejido blando & Beta 539738 & 1530 & 30 & 1 & 2 \\
\hline Guayadeque & Tejido blando & Beta 539733 & 1420 & 30 & 1 & 2 \\
\hline Guayadeque & Tejido blando & Beta 565464 & 980 & 30 & 1 & 2 \\
\hline Guayadeque & Hueso humano & Beta 565474 & 1110 & 30 & 1 & 2 \\
\hline Guayadeque & Hueso humano & DAMS 032113 & 1340 & 34 & 1 & 2 \\
\hline Guayadeque & Hueso humano & DAMS 032114 & 853 & 25 & 1 & 2 \\
\hline Guayadeque & Hueso humano & DAMS 032115 & 927 & 23 & 1 & 2 \\
\hline Guayadeque & Hueso animal & Beta 510720 & 1340 & 30 & 1 & 2 \\
\hline Guayadeque & Tejido blando & Beta 565459 & 1460 & 30 & 1 & 2 \\
\hline Guayadeque & Hueso humano & Beta 565460 & 990 & 30 & 1 & 2 \\
\hline Guayadeque & Tejido blando & Beta 565461 & 1430 & 30 & 1 & 2 \\
\hline
\end{tabular}




\begin{tabular}{|c|c|c|c|c|c|c|}
\hline Guayadeque & Hueso humano & Beta 565462 & 1210 & 30 & 1 & 2 \\
\hline Guayadeque & Piel mortaja & Beta 565476 & 920 & 30 & 1 & 2 \\
\hline Hormiguero & Hueso humano & Beta-302332 & 950 & 30 & 1 & 1 \\
\hline Hormiguero & Hueso humano & $1 \mathrm{~A} 1070$ & 940 & 30 & 1 & 1 \\
\hline Hormiguero & Hueso humano & 1A1071 & 1005 & 30 & 1 & 1 \\
\hline Hormiguero & Hueso humano & 1A1072 & 780 & 30 & 1 & 1 \\
\hline Hormiguero & Hueso humano & SacA500013 & 920 & 30 & 1 & 1 \\
\hline Hormiguero & Hueso humano & SacA500014 & 985 & 30 & 1 & 1 \\
\hline Hormiguero & Hueso humano & SacA500015 & 975 & 30 & 1 & 1 \\
\hline Isleta & Hueso humano & Beta 510719 & 1120 & 30 & 2 & 1 \\
\hline Isleta & Hueso humano & Beta 565470 & 1000 & 30 & 2 & 1 \\
\hline Isleta & Hueso humano & Beta 565471 & 1000 & 30 & 2 & 1 \\
\hline Candelarias & Hueso humano & - & 540 & 30 & 3 & 1 \\
\hline Candelarias & Hueso humano & Beta 315247 & 360 & 30 & 3 & 1 \\
\hline Candelarias & Hueso humano & Beta 218613 & 560 & 50 & 3 & 1 \\
\hline Candelarias & Hueso humano & Beta 216161 & 760 & 40 & 3 & 1 \\
\hline Candelarias & Hueso humano & Beta 216162 & 590 & 40 & 3 & 1 \\
\hline Caserones & Hueso humano & - & 690 & 40 & 3 & 1 \\
\hline Lomo Galeón & Hueso humano & Beta 302333 & 730 & 30 & 3 & 1 \\
\hline Huesas & Hueso humano & Beta 510712 & 1190 & 30 & 1 & 1 \\
\hline Huesas & Hueso humano & Beta 510713 & 1270 & 30 & 1 & 1 \\
\hline Huesas & Hueso humano & Beta 510711 & 1210 & 30 & 1 & 1 \\
\hline Maipés & Hueso humano & Beta 297168 & 1210 & 30 & 2 & 1 \\
\hline Maipés & Hueso humano & Beta 297170 & 1180 & 30 & 2 & 1 \\
\hline Maipés & Hueso humano & Beta 297171 & 1220 & 30 & 2 & 1 \\
\hline Maipés & Hueso humano & Beta 297172 & 1250 & 30 & 2 & 1 \\
\hline Maipés & Tejido vegetal & Beta 384699 & 1220 & 30 & 2 & 1 \\
\hline Maipés & P.dental humana & Beta 384700 & 1210 & 30 & 2 & 1 \\
\hline Maipés & Hueso humano & Beta 510714 & 1050 & 30 & 2 & 1 \\
\hline Metropole & Hueso humano & Beta 32663 & 540 & 70 & 3 & 1 \\
\hline Metropole & Hueso humano & Beta 539742 & 590 & 30 & 3 & 1 \\
\hline Amadores & Hueso humano & Beta-315249 & 1000 & 30 & 1 & 1 \\
\hline Rosiana & Hueso humano & DAMS 032112 & 1205 & 25 & 1 & 2 \\
\hline Juan Primo & Hueso humano & Beta 248144 & 710 & 40 & 3 & 1 \\
\hline Juan Primo & Hueso humano & Beta 248145 & 800 & 50 & 3 & 1 \\
\hline Juan Primo & Hueso humano & Beta 248149 & 660 & 40 & 3 & 1 \\
\hline Juan Primo & Hueso humano & Beta 248146 & 640 & 40 & 3 & 1 \\
\hline Juan Primo & Hueso humano & Beta 248147 & 630 & 40 & 3 & 1 \\
\hline Juan Primo & Hueso humano & Beta 248148 & 580 & 40 & 3 & 1 \\
\hline Maspalomas & Hueso humano & Beta 365839 & 610 & 30 & 3 & 1 \\
\hline Maspalomas & Hueso humano & Beta 365840 & 440 & 30 & 3 & 1 \\
\hline Maspalomas & Hueso humano & Beta 210778 & 610 & 70 & 3 & 1 \\
\hline Maspalomas & Hueso humano & Beta 210779 & 820 & 40 & 3 & 1 \\
\hline
\end{tabular}




\begin{tabular}{|c|c|c|c|c|c|c|}
\hline Maspalomas & Hueso humano & Beta 302334 & 550 & 30 & 3 & 1 \\
\hline Maspalomas & Hueso humano & Beta 210779 & 820 & 40 & 3 & 1 \\
\hline Maspalomas & Hueso humano & SacA50008 & 670 & 30 & 3 & 1 \\
\hline Maspalomas & Hueso humano & SacA50009 & 640 & 30 & 3 & 1 \\
\hline Maspalomas & Hueso humano & SacA50010 & 675 & 30 & 3 & 1 \\
\hline Maspalomas & Hueso humano & SacA50011 & 635 & 30 & 3 & 1 \\
\hline Maspalomas & Hueso humano & SacA50012 & 695 & 30 & 3 & 1 \\
\hline Pozo Izquierdo & Hueso humano & DAMS 0179 & 757 & 27 & 3 & 1 \\
\hline Antigafo & Hueso humano & Beta 272295 & 710 & 40 & 3 & 1 \\
\hline Antigafo & Hueso humano & Beta 272294 & 420 & 40 & 3 & 1 \\
\hline Antigafo & Hueso humano & Beta 272293 & 460 & 40 & 3 & 1 \\
\hline Silva & Hueso humano & Beta 565468 & 1000 & 30 & 1 & 1 \\
\hline Silva & Hueso humano & Beta 565482 & 800 & 30 & 1 & 1 \\
\hline S_Pinillo & Hueso humano & Beta 565480 & 990 & 30 & 1 & 2 \\
\hline Tederas & Hueso humano & DAMS 32111 & 542 & 28 & 3 & 2 \\
\hline Tabacalete & Hueso humano & SacA500025 & 1300 & 30 & 1 & 2 \\
\hline Tabacalete & Hueso humano & SacA500026 & 1375 & 30 & 1 & 2 \\
\hline Tabacalete & Hueso humano & SacA500345 & 1270 & 30 & 1 & 2 \\
\hline Tabacalete & Hueso humano & SacA500346 & 1315 & 30 & 1 & 2 \\
\hline Tabacalete & Hueso humano & SacA500347 & 1240 & 30 & 1 & 2 \\
\hline Tabacalete & Hueso humano & SacA500348 & 1285 & 30 & 1 & 2 \\
\hline Tirajana & Hueso humano & Beta 539740 & 1240 & 30 & 1 & 2 \\
\hline Tirajana & Hueso humano & Beta 565469 & 1300 & 30 & 1 & 2 \\
\hline
\end{tabular}

Tabla 1. Dataciones radiocarbónicas incorporadas al estudio. Tipo: (1) cueva; (2) túmulo; (3) fosa/cista. Territorio: (1) $<250 \mathrm{msnm}$; (2) >250 msnm

\section{Más información sobre las dataciones en}

https://dataciones.grancanaria.com/

http://www.elmuseocanario.com/images/documentospdf/piezadelmes/2017/piezanoviembre2017.pdf http://www.elmuseocanario.com/images/documentospdf/piezadelmes/2019/piezamarzo2019.pdf http://www.elmuseocanario.com/images/documentospdf/piezadelmes/2020/piezafebrero2020.pdf http://anuariosatlanticos.casadecolon.com/index.php/aea/article/view/10530 